\title{
Guidelines for biomarkers in autoimmune rheumatic diseases - evidence based analysis
}

\author{
Roberto Giacomellia, *, Antonella Afeltra ${ }^{\mathrm{b}}$, Alessia Alunno ${ }^{\mathrm{c}}$, Elena Bartoloni-Bocci ${ }^{\mathrm{c}}$, Onorina Berardicurti ${ }^{\mathrm{a}}$, \\ Michele Bombardieri ${ }^{\mathrm{d}}$, Alessandra Bortoluzzi ${ }^{\mathrm{e}}$, Roberto Caporalif ${ }^{\mathrm{f}}$, Francesco Caso ${ }^{\mathrm{g}}$, Ricard Cervera ${ }^{\mathrm{h}}$,

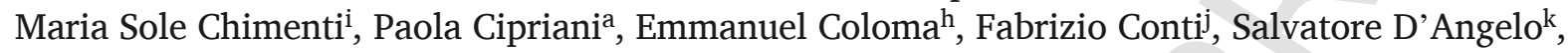
Salvatore De Vital, Salvatore Di Bartolomeo ${ }^{\mathrm{a}}$, Oliver Distler ${ }^{\mathrm{m}}$, Andrea Doria ${ }^{\mathrm{n}}$, Eugen Feist ${ }^{\mathrm{o}}$, Benjamin A. Fisher ${ }^{\mathrm{p}}$, Maria Gerosa ${ }^{\mathrm{q}}$, Michele Gilio ${ }^{\mathrm{k}}$, Giuliana Gugginor ${ }^{\mathrm{r}}$, Vasiliki Liakoulia , Domenico Paolo Emanuele Margiotta ${ }^{\mathrm{b}}$, Pierluigi Meroni $^{q}$, Gianluca Moroncinis ${ }^{\mathrm{s}}$, Federico Perosa ${ }^{\mathrm{t}}$, Marcella Prete ${ }^{\mathrm{t}}$, Roberta Priori ${ }^{\mathrm{j}}$, Chiara Rebuffi ${ }^{\mathrm{u}}$, Piero Ruscitti ${ }^{\mathrm{a}}$, Raffaele Scarpa ${ }^{\mathrm{g}}$, Yehuda Shoenfeld ${ }^{\mathrm{v}}$, Monica Todoerti ${ }^{\mathrm{f}}$, Francesco Ursini ${ }^{\mathrm{w}}$, Guido Valesini ${ }^{\mathrm{j}}$, Serena Vettori ${ }^{\mathrm{x}}$, Claudio Vitali ${ }^{\mathrm{y}}$, Athanasios G. Tzioufas ${ }^{\mathrm{z}}$

\footnotetext{
a Department of Biotechnological and Applied Clinical Science, Rheumatology Unit, School of Medicine, University of L'Aquila, Delta 6 Building, Via dell'Ospedale, 67100 L'Aquila, Italy

${ }^{\mathrm{b}}$ Department of Medicine, Unit of Allergology, Immunology, Rheumatology, Campus Bio-Medico University of Rome, Via Álvaro del Portillo 21,00128 Rome, Italy

${ }^{\mathrm{c}}$ Rheumatology Unit, Department of Medicine, University of Perugia, Perugia, Italy

${ }^{\mathrm{d}}$ William Harvey Research Institute, Barts and The London School of Medicine and Dentistry, London, UK

e Department of Medical Science, Section of Rheumatology, University of Ferrara and Azienda Ospedaliero-Universitaria S.Anna, Cona, Ferrara, Italy

${ }^{\mathrm{f}}$ IRCCS Policlinico San Matteo Foundation, Division of Rheumatology, University of Pavia, Pavia, Italy

${ }^{g}$ Department of Clinical Medicine and Surgery, Rheumatology Unit, University of Naples Federico II, Naples, Italy

${ }^{\text {h }}$ Department of Autoimmune Diseases, Hospital Clínic, Barcelona, Catalonia, Spain

${ }^{\mathrm{i}}$ Department of Medicina dei Sistemi, Rheumatology, Allergology and Clinical Immunology, University of Rome Tor Vergata, Rome, Italy

${ }^{j}$ Department of Internal Medicine and Medical Specialties, Rheumatology Unit, Sapienza University of Rome, Rome, Italy

${ }^{\mathrm{k}}$ Rheumatology Institute of Lucania (IReL) - Rheumatology Department of Lucania, "San Carlo" Hospital of Potenza and "Madonna delle Grazie" Hospital of Matera, Potenza, Italy

${ }^{1}$ Department of Medical and Biological Sciences, Rheumatology Clinic, Azienda Ospedaliero Universitaria S. Maria della Misericordia, University of Udine, Udine, Italy

${ }^{m}$ Department of Rheumatology, University Hospital Zurich, Zurich, Switzerland

${ }^{n}$ Department of Medicine, DIMED, Division of Rheumatology, University of Padua, Padua, Italy

${ }^{\circ}$ Department of Rheumatology and Clinical Immunology of the Charité, Universitätsmedizin Berlin, Berlin, Germany

${ }^{\mathrm{p}}$ Rheumatology Research Group and Arthritis Research UK Rheumatoid Arthritis Pathogenesis Centre of Excellence (RACE), University of Birmingham, Birmingham, UK; Department of

Rheumatology, University Hospitals Birmingham NHS Trust, Birmingham, UK

${ }^{\mathrm{q}}$ Lupus Clinic, Division of Rheumatology, ASST Pini, Department of Clinical Sciences and Community Health, University of Milan, Italy

${ }^{\mathrm{r}}$ Rheumatology Section, Department of Internal Medicine, University of Palermo, Palermo, Italy;

${ }^{s}$ Department of Clinical and Molecular Sciences, Università Politecnica delle Marche, Ancona, Italy

${ }^{\mathrm{t}}$ Department of Biomedical Sciences and Human Oncology (DIMO), Systemic Rheumatic and Autoimmune Diseases Unit, University of Bari Medical School, Bari, Italy

u Grant Office and Scientific Documentation Center, IRCCS Policlinico San Matteo Foundation, Pavia, Italy

${ }^{v}$ Zabludowitz Centre for Autoimmune Diseases, Sheba Medical Centre, Tel-Hashomer, Israel

${ }^{\mathrm{w}}$ Department of Health Sciences, University of Catanzaro "Magna Graecia", Catanzaro, Italy

${ }^{x}$ Department of Clinical and Experimental Medicine, Rheumatology Section, Second University of Naples, Naples, Italy

${ }^{\text {y }}$ Villa San Giuseppe, Istituto S. Stefano, Como, Italy

${ }^{\mathrm{z}}$ Pathophysiology Department, General Hospital of Athens "Laiko", Medical School, National and Kapodistrian University of Athens, Greece
}

\section{ART ICLE INFO}

\section{Keywords:}

Biomarker

Rheumatoid arthritis

Spondyloarthritides

Systemic sclerosis

Systemic lupus erythematosus

Antiphospholipid syndrome

Sjogren's syndrome

\section{A B S T R A C T}

Autoimmune rheumatic diseases are characterised by an abnormal immune system response, complement activation, cytokines dysregulation and inflammation. In last years, despite many progresses in managing these patients, it has been shown that clinical remission is reached in less than $50 \%$ of patients and a personalised and tailored therapeutic approach is still lacking resulting in a significant gap between guidelines and real-world practice. In this context, the need for biomarkers facilitating early diagnosis and profiling those individuals at the highest risk for a poor outcome has become of crucial interest. A biomarker generally refers to a measured characteristic which may be used as an indicator of some biological state or con-

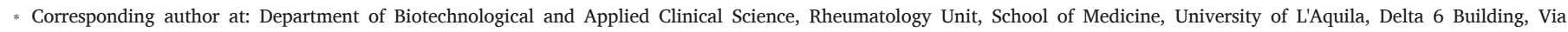
dell'Ospedale, 67100 L'Aquila, Italy.

Email address: roberto.giacomelli@cc.univaq.it (R. Giacomelli) 
dition. Three different types of medical biomarkers has been suggested: i. mechanistic markers; ii. clinical disease markers; iii. therapeutic markers. A combination of biomarkers from these different groups could be used for an ideal more accurate diagnosis and treatment. However, although a growing body of evidence is focused on improving biomarkers, a significant amount of this information is not integrated on standard clinical care.

The overarching aim of this work was to clarify the meaning of specific biomarkers during autoimmune diseases; their possible role in confirming diagnosis, predicting outcome and suggesting specific treatments.

\section{Introduction}

Autoimmune rheumatic diseases are characterised by an abnormal immune system response, complement activation, cytokines dysregulation and inflammation [1]. These heterogeneous disorders may affect various organs, and although their clinical presentations may be different, these diseases share significant genetic risk factors and common regulatory mechanisms [2]. Environmental and female-associated factors also play pathogenic roles in development of autoimmune diseases $[1,2]$. In last years, despite many progresses in managing these patients, it has been shown that clinical remission is reached in less than $50 \%$ of patients and a personalised and tailored therapeutic approach is still lacking resulting in a significant gap between guidelines and real-world practice $[3,4]$. In this context, the need for biomarkers facilitating early diagnosis and profiling those individuals at the highest risk for a poor outcome has become of crucial interest [5,6]. A biomarker generally refers to a measured characteristic which may be used as an indicator of some biological state or condition [7]. Three different types of medical biomarkers have been suggested: i. mechanistic markers; ii. clinical disease markers; iii. therapeutic markers [8]. In the first group, subcellular changes may lead to alterations in proteins detectable as biomarkers and reflecting the ongoing cellular process and manipulated signalling pathways [9]. In the second group, the disease development is associated with the changes in proteins that are detected by proteomics, defining the clinical disease markers, which indicate state of progression, severity and location of the disease [10]. In the third group, the treatment of a disease may point out therapeutic biomarkers, associated with a specific changing pattern after the drug administration [11]. Taking together these observations, a combination of biomarkers from these different groups could be used for an ideal more accurate diagnosis and treatment. However, although a growing body of evidence is focused on improving biomarkers [7-11], a significant amount of this information is not integrated on standard clinical care. (See Table 1.)

The overarching aim of this work was to clarify the meaning of specific biomarkers during autoimmune diseases; their possible role in confirming diagnosis, predicting outcome and suggesting specific treatments.

\section{Methods}

\subsection{Aims of the project}

The overarching aim of this workshop is to clarify the meaning of specific biomarkers during autoimmune diseases; their possible role in confirming diagnosis, predicting outcome and suggesting specific treatments. The general methodology based on a Delphi Technique-based aimed at producing, starting from the results of a systematic review of available literature, a set of statements summarising the consensus among the Experts, as previously reported [12]. This systematic review has been designed to be included in an International project named "Clinical and biological biomarkers in conventional and/or biological therapies. From pathology to treatment: what evidence in
Table 1

\begin{tabular}{lc}
\hline Statements & LoE \\
\hline
\end{tabular}

RA Working Group

In a population-based setting, higher level of anti-cyclic citrullinated peptide (anti-CCP), defined as $\geq 3$-fold the ULN, might be more clinically useful for RA diagnosis.

$\mathrm{RF}$ positivity together with very high levels of aCCP might be useful to discriminate RA patients from other rheumatic diseases.

High levels of anti-CCP and/or RF seem to correlate with higher disease activity and worse radiographic progression over time. High titre aCCP and $\mathrm{RF}$ are correlated with better response to rituximab, while only very high aCCP titers seem to be associated with better response to abatacept.

Anti-CarP antibodies may serve as predictors of more severe radiographic progression in RA and potentially associated with more severe disease course

In RA, the possible role of MBDA in predicting disease course and response to treatments is still controversial.

SpA Working Group

Although enthesitis may be considered a clinical marker of PsA, there is no evidence showing that its presence at baseline predicts the response to different bDMARDs (TNFis, IL17is, IL12/IL23is).

Although data available on biomarkers for predicting therapeutic response in $\mathrm{SpA}$ are scarce, CRP in clinical practice may be useful in predicting TNFis response.

SSc working group

Anti-topoisomerase 1 antibody is a biomarker for faster progression of SSc ILD

KL-6, SP-D, CCL18 may be considered as biomarkers for short-term progression of SSc-ILD.

The DETECT algorithm is useful to identify SSc patients at higher risk for PAH who should undergo RHC.

High levels of plasma CXCL4 may predict development of PAH in SSc patients.

High levels of serum Anti-AT1R and Anti-ETAR antibodies may predict development of PAH in SSc.

SLE working group

There is no evidence that a negative serology predicts a successful withdrawal of therapy in SLE patients (with or without nephritis) in clinical remission.

In lupus nephritis, negative serology does not predict a successful withdrawal of therapy.

APS working group

The addition of anti-domain I to laboratory classification criteria seems to increase the risk of thrombosis.

There is no evidence that anti-domain I can substitute anti-beta2GPI I.

The presence of anti-PS/PT seems to increase the risk of clinical manifestations of APS.

The presence of anti-PS/PT seems to identify very few patients with the so called seronegative APS

pSS working group

The predictive value of CXCL13 in pSS, for both poor prognosis and therapeutic response, remains to be clarified.

The predictive value of baseline BAFF levels for lymphoma development and therapeutic response to RTX should be further assessed.

The clinical utility of the presence of GC-like structures, in MSGs, to predict lymphoma development should be further assessed with standardization of technique and multicentre studies given the relatively low incidence of lymphoma.

rheumatic and autoimmune diseases? 2th International workshop" aimed to update some features in management of patients affected by autoimmune disease. As a part of an International project, a Scientific Committee composed by a group of experts and bibliographic fellows identified some relevant clinical questions on biomarkers in autoimmune diseases, needing further and updated clarifications according 
to available scientific evidence and joined Experts' opinion. These invitations were a consequence of the individuals' contributions to the specific fields included in the topics of the meeting as well as deliberations among members of the steering committee. Six autoimmune diseases were selected and evaluated: rheumatoid arthritis (RA), spondyloarthritides (SpA), systemic sclerosis (SSc), systemic lupus erythematosus (SLE), antiphospholipid syndrome (APS), primary Sjogren's syndrome (pSS). These topics were developed and updated throughout an extensive bibliographic review by the Steering Board, after joining common limits and methods of search. For each selected topic, preliminary statements based on available scientific results have been presented in accordance with their level of evidence, discussed, eventually reformulated, and voted through a Delphi-method during a Consensus involving a panel of International Experts. Statements supported by $\geq 66 \%$ of votes were accepted as final statements, while the others were rejected outright. This project has been concluded in Italy on October 6-7, 2017.

\subsection{Search design}

For each of these 6 topics, a systematic literature search was performed in indexed international Journals (Medline via Pubmed, Scopus, Cochrane database). The Scientific Committee decided to analyse the literature from July 1, 2007 - July 1, 2017. The choices of temporal limits, online databases and methodology were originally discussed and shared by participants in order to gain homogeneous results.

\subsection{Search strategy}

The search strategy combined indexed and free-text terms, interventions and outcomes of interest in Medline via Pubmed, Scopus, Cochrane database, as requested for each single topic. PICO strategy was also joined as shared rephrasing strategy across working groups, along with pre-defined "Population", "Intervention", "Comparison", "Outcomes", as requested by single topic research question. The main search was thus formulated using a string of relevant terms of research. In addition, the main keywords were used in different combinations in order to improve the sensitivity of the search strategy. The bibliography of relevant articles was also hand-searched for identification of other potentially suitable studies.

\subsection{Eligibility criteria}

Included studies were full-text manuscripts in English language conducted in adult patients with autoimmune diseases. To be included in the final analysis, studies had to meet the following joined inclusion criteria: 1) study design: systematic review and meta-analysis, randomised controlled trial (RCT), quasi-RCT (trials in which allocation to treatment was made by alternation, use of alternate medical records, date of birth or other expected methods), observational cohort studies or case series; 2) data concerning population, intervention, comparison and outcomes were requested for each single selected topic. Narrative reviews, editorials, scientific conference abstracts, case reports and pre-clinical studies have been excluded from the purpose of this work. Papers retrieved by literature search but reporting insufficient data according to selected PICO strategy were excluded from the review. The hierarchy of study types was indicated by levels of evidence suggested by Oxford University (http://www.cebm.net/ oxford-centre-evidence-based-medicine-levels-evidence-march-2009/).

\subsection{Study identification and data extraction}

In each group working on a single topic, full-text articles were screened and selected analysing titles and abstracts by bibliographic fellows, then independently verified by corresponding senior reviewers. After the screening phase, the bibliographic fellows also independently evaluated the selected abstracts and the full-text of these studies to determine eligibility according to the eligibility criteria. Any uncertainties and/or disagreements were resolved by discussion until reaching a final consensus. Data extraction was also performed by bibliographic fellows and independently verified by corresponding senior reviewers. After that, the results of the analysis of literature were summarised, presented, and further inputs were obtained from expanded working groups with other authors. Conflicting results were analysed by discussion taking into account quality of assessed studies until reaching an agreement into the single working group. The statements were thus formulated according to results and quality of evaluated works. Further disagreements were resolved by discussion until reaching a final consensus. In the subsequent plenary session, the statements were subjected to be voted as 'yes' (agreement) or 'no' (disagreement) from the entire panel of Experts. Statements supported by $\geq 66 \%$ of positive votes were accepted while the others were rejected outright. At this final stage, only suggestions for improvements of clarity of wording or addressing redundancies were considered, while any change to the meaning was not accepted.

\section{RA working group}

To date, recognition and better definition of disease-specific biomarkers, easily and routinely detectable in patients serum samples, could be relevant for diagnostic and prognostic purposes in the view of a more patients' tailored approach, regardless of the underlying condition. Anti-citrullinated protein antibodies (ACPA) and rheumatoid factor (RF) are well recognized clinically relevant biomarkers in rheumatoid arthritis (RA) patients [13]. Their pathogenic role has been well characterized, being detectable in the serum in pre-clinical phase many years before clinical presentation of the disease. Moreover, both biomarkers have been included in the new 2010 RA classification criteria into a score-based algorithm where both qualitative (negative versus positive) and quantitative (low level versus high level) evaluations have been inserted, with high level (predefined as $\geq 3$ times the ULN for the laboratory test and assay) having greater weight in substantially contributing to RA diagnosis [14]. In the 2016 update of EULAR recommendations for RA management, either presence and levels of RF and/or ACPA have been listed among several other prognostic factors to be considered during therapeutic management, too. Moving from this starting point, it could be clarified whether a better definition of such biomarkers (high versus low serum levels) might help clinicians in term of discriminative ability in diagnostic accuracy (as diagnostic and differential diagnostic tool) and prognostic stratification (disease course, radiographic damage, response to therapy). In other words, throughout an extended systematic review on the topic in line with joined pre-specified limits and settings, the clinical meaning of "level criterion" of "serology" item has been investigated in light of diagnostic and prognostic purposes (see corresponding PICOs rephrasing in Table 1/in the attached files). Any case, when interpreting lab results of RA serum biomarkers, several concerns might be taken into account justifying contrasting results across studies: lack of harmonization and standardization of RF and ACPA tests (different methods, cut-off levels, reference materials); assessment of different RF isotypes (IgG, IgA, IgM) and ACPA specificities; disease and demographical characteristics of enrolled RA patients (ethnic and genetic background of target 
populations, disease duration, environmental exposition); lastly, when considering the impact of biomarkers on prognosis, previous and current treatment itself might be a confounding factor, too. Moreover, as a further source of heterogeneity and variability, different definitions of high and low levels of either RF and/or ACPA have been proposed across studies, mostly in accordance to EULAR/ACR 2010 classification criteria (3 times higher the cut off values) [14].

\subsection{Diagnostic purposes, the role of anti-CCP}

3.1.1. In a population-based setting, higher level of anti-cyclic citrullinated peptide (anti-CCP), defined as $\geq 3$-fold the ULN, might be more clinically useful for RA diagnosis. LoE $2 b$

Two population-based studies assessed in large unselected cohorts the discriminatory capacity of anti-CCP in diagnosing RA for either prevalent (cross-sectional analysis) and incident (prospective analysis) cases $[15,16]$. These studies offered the unique advantage of testing such biomarker in numerous healthy (independently of any previous/ current sings and/or symptoms of arthritis or any previous lab measurement) rather than selected subjects, like blood donors or high-risk populations (symptom-free first-degree relatives of RA patients, patients with arthralgia, patients suffering from other autoimmune diseases). In both studies, high anti-CCP levels have been defined in accordance to ACR/ EULAR 2010 classification criteria. In the study by Hensvold, high versus low anti-CCP2 titres were associated with increased specificity for prevalent RA with only minor decrease in sensitivity (98\% and $66 \%$ and $99 \%$ and $62 \%$ with positive anti-CCP2 and high anti-CCP2 cut-off, respectively). In the study by Demourelle et al [15], when prevalent cases were considered (established RA patients), the higher the adopted anti-CCP cut-off levels, the better the specificity (93.1\% using standard cut-off level, $97.2 \%$ with $\geq 3 \mathrm{X}$ standard cut-off level for anti-CCP3 assay). Despite this, it remains debatable whether a single assay with a common threshold could be considered optimal for all clinical settings.

\subsection{2. $R F$ positivity together with very high levels of anti-CCP might be} useful to discriminate RA patients from other rheumatic diseases. LoE 3

We identified 6 research articles that compared anti-CCP test in term of discriminative diagnostic performance in patients suffering from RA or other RA-mimicking rheumatic or non-rheumatic diseases [17-22]. All of them have case-control design involving as control population patients with non-RA inflammatory and non-inflammatory rheumatic diseases (osteoarthritis, systemic lupus erythematosus, Sjogren's syndrome, spondyloarthritis, systemic sclerosis, crystal-induced arthritis, infectious arthritis, systemic sclerosis, fibromyalgia, and/or other related conditions) [17-22]. The underlying hypothesis is that elevated anti-CCP levels, as more specific RA markers respect to RF, can help in better supporting the differential diagnosis of RA and the above indications. In the largest study by Pietrapertosa, specificity and sensitivity of anti-CCP2 antibodies measured by ELISA were tested in 787 patients with RA, 1024 patients with other autoimmune/inflammatory rheumatic disease and 401 subjects without autoimmune rheumatic diseases (osteoarthritis and fibromyalgia) [22].Using ROC curve analysis, the cut-off value of $2.8 \mathrm{U} / \mathrm{mL}$ for anti-CCP2 had the highest diagnostic accuracy for distinguishing between RA patients and both control cohorts, but a value of anti-CCP2 $15 \mathrm{U} / \mathrm{mL}$ ( 3 fold the manufacturer cut-off) was associated with a significant increase in the likelihood of RA disease. In addition, in a Chinese population, the combination of high titre anti-CCP antibodies ( $\geq 100 \mathrm{RU} / \mathrm{ml}$ ) with a concomitant positive RF test exhibited the greatest diagnostic specificity, especially in the early stage of the disease, respect to single positive RF and/or anti-CCP or double positive or RF + and low positive anti-CCP [20]. Contrasting results come from a French and Korean studies, where anti-CCP level (high versus levels) did not offer further information in discriminating RA from other non-RA diseases: anti-CCP positivity resulted significantly more prevalent in RA patients, whereas high titres anti-CCP, when positive, did not substantially differ across rheumatic RA and non-RA conditions $[18,21]$.

\subsection{Prognostic purposes, $R F$ and anti-CCP}

High levels of anti-CCP and/or RF seem to correlate with higher disease activity and worse radiographic progression over time. High titre anti-CCP and RF are correlated with better response to rituximab, while only very high anti-CCP titres seem to be associated with better response to abatacept. LoE $2 b$.

\subsubsection{Radiographic progression}

Globally, high levels of either RF and/or anti-CCP were significantly associated with worst deterioration over time in RA patients [23-31]. In the study by Syversen et al. among 125 established RA patients, anti-CCP considered either as categorical and quantitative parameter was significantly associated with radiographic progression according to the van der Heijde modified Sharp score of the hands at 10-year assessment [23]. Anti-CCP (OR 4.0; 95\% CI 1.6 to 10.0) was the strongest independent predictor of radiographic progression, in a level-dependent fashion: respect to anti-CCP-negative patients, low to moderate (25-200 $\mathrm{U} / \mathrm{ml}$ ) and high levels ( $>200 \mathrm{U} / \mathrm{ml}$ ) anti-CCP subjects were more likely to develop radiographic progression over time (corresponding Odds Ratios/OR being $2.6,95 \%$ CI 0.9 to 7.2 and $9.9,95 \%$ CI 2.7 to 36.7 , respectively). Higher cut-off RF levels did not change the model, without improving or weakening the prognostic effect of anti-CCP. Conversely, in a cohort of Japanese early RA patients (disease duration $<2$ years), median total Sharp score at 2 years did not significantly differ among baseline low-titre (median score [IQR] 23.0 [8.0, 47.3]) versus high-titre (21.5 [7.5, 52.0]) anti-CCP groups; nevertheless both subgroups substantially differed respect to anti-CCP negative patients (2-year median Sharp score [IQR] $6.0[2.0,12.0], \mathrm{p}=0.00001$ versus low- anti-CCP $\mathrm{p}=0.00001$ versus high-anti-CCP ) [27]. Different target populations (established versus early disease in divergent ethnic background), outcome time-points (10-year versus 2-year), anti-CCP cut-off pre-defined levels, might account partly for divergent results between these 2 studies [30]. A level-dependent effect on structural outcome was confirmed for RF-titres too, in line with Van der Linden et al. study [25]. Regardless of the given definition, high RF levels (defined as $>3$ cut-off value, as in the 2010 ACR/EULAR classification criteria or RF $>50$ units $/ \mathrm{ml}$ / $\mathrm{RF50}$, in line with definition given by previous studies) were significantly associated with a higher risk of radiographic progression over 7 years in 686 RA patients; nevertheless the association between high RF level and RA severity was not as strong as that between anti-CCP positivity and RA severity, with significantly greater progression rate for ACPA + versus both high RF levels definitions. Similarly, pooled data from five clinical trials demonstrated a significant RF level-dependent effect $(\mathrm{RF}-, \mathrm{RF}$ low,$+ \mathrm{RF}$ high + ) on radiographic damage (proportion of progressors and of rapid progressors) in the study by Aletaha et al. after adjustment for relevant and confounding parameters like disease duration, baseline damage, baseline CRP and baseline ESR [28]. In addition, even in case of DMARDs-induced disease control, high level ACPA ( $>48 \mathrm{U} / \mathrm{mL}$, as defined through ROC curve) resulted the strongest independent predictor of clinically relevant radiographic progression in a real-world Japanese prospective study. Possibly, an additive effect between ACPA and RF could be taken into consideration when considering RA structural damage: only in ACPA positive RA patients, TC-detected erosion burden at hands (number and size of erosions) was cross-sectional associated with RF-levels; thus, RF might act as a 
structural damage enhancer only in ACPA-positive patients in a dose-dependent manner [26].

\subsubsection{Disease activity}

Higher anti-CCP and RF concentrations resulted associated with greater disease activity over time along with lower chance to achieve sustained remission in men with established RA mainly under csDMARDs background [32,33]. Similarly, baseline low RF and anti-CCP levels (arbitrarily chosen by the authors) were independently predictive of clinical remission and low disease activity achievement at 12 months in 90 Romanian RA patients starting their first TNFi. High anti-CCP titres significantly correlated with higher RF levels, DAS28 score and more severe morning stiffness duration in a Chinese population by Li et al [20].

\subsubsection{Response to therapy}

The systematic review by Salgado included two studies considering the impact of RF levels (high versus low) on TNFi response: only the one by Klaasen er al enter our review due to publication date [34,35]. Among 101 RA patients eligible to infliximab, presence and high ( $>100$ $\mathrm{UI} / \mathrm{mL}$ ) levels of RF-IgM significantly and positively correlate with primary response to therapy at 16 weeks, while high RF-IgG and RF-IgA did not. On the contrary, in the study by Salgado no significant differences across 50th, 25th and 75th percentiles of baseline IgM RF titres were observed between responders and non-responders (EULAR response criteria at 24 weeks) in a combined cohort of 3 Spanish centres experience [36]. Such contrasting results for RF levels and TNFi response are in line with previous published reports on the topic [37]. High levels of aCCP ( $>400 \mathrm{UI} / \mathrm{mL}$ ) resulted significantly associated with greater probability of achieving ACR20 response after 16 weeks of adalimumab medication in a cohort of 70 RA patients [38]. Similarly, among 108 RA patients those with baseline anti-CCP titres $>300 \mathrm{UI} / \mathrm{mL}$ were more likely (more than 3 times) to gain major EULAR response 6 months after the first rituximab cycle after failure of at least one TNFi in the retrospective analysis by Narvaez et al. [39]. Finally, from the post-hoc analysis of the AMPLE trial as a head-to-head comparison between adalimumab and abatacept on a MTX background in MTX-IR patients, baseline anti-CCP2 positivity was associated with a better clinical response to both $\mathrm{ABA}$ and $\mathrm{ADA}$. Nevertheless, only in ABA group, patients with the highest (fourth quartile, corresponding to very high levels, 1060-4894 AU/mL) vs lower CCP2 concentrations (Q1) had better clinical response with ABA; this association was not observed in ADA group. Despite not designed and powered for such comparison, there was no inter-group difference in term of clinical response between the highest quartile group patients belonging to $\mathrm{ADA}$ or $\mathrm{ABA}$ group [40].

In RA patients, auto-antibodies like RF and ACPA represent the hallmark of the break of immune tolerance, accompanying and justifying both the onset and the burden of the disease itself. Very recently, the "level criterion" of such biomarkers entered into both classification and prognostic stratification criteria. In line with retrieved scientific evidences, joined by an international panel of expert rheumatologists, a better definition of either RF and ACPA in term of high versus low levels might add relevant information for diagnostic and prognostic purposes, thus supporting clinicians for a more specific disease recognition and prognostic stratification. To date, efforts should be pointed out to harmonize and standardize biomarkers detection in order to allow comparison and generalization of results either at a research level, and in the everyday clinical setting.

\subsection{Anti-CarP in predicting more severe $R A$ course}

\subsubsection{Anti-CarP antibodies may serve as predictors of more severe} radiographic progression in $R A$ and potentially associated with more severe disease course. LoE $2 b$

Antibodies against carbamylated proteins (anti-CarP antibodies), recognising proteins post-translationally modified by a process of carbamylation, have been recently detected in rheumatoid arthritis (RA) [41-43]. In this systematic review of literature, we investigated the prognostic role of anti-CarP antibodies in predicting more severe RA course. After screening titles and abstracts, 5 observational studies were retrieved and included in the review [44-48].

Shy et al assessed anti-CarP in sera from 571 RA patients by Leiden EAC cohort and 305 healthy controls [44]. Authors reported 45\% of RA patients were positive for IgG anti-CarP antibodies and $43 \%$ positive for IgA anti-CarP antibodies. Furthermore, anti-CarP antibodies were associated with a more severe radiographic damage over 7 years, analysing all enrolled patients. Of note, anti-CarP IgG antibodies were associated with a more severe radiological progression also in ACPA-negative RA [44]. These findings have been replicated in subsequent studies, assessing anti-CarP antibodies to be an independent predictor of radiographic progression $[45,46]$. Ajeganova et al. assessed 576 Swedish and 628 Dutch patients and observed anti-CarP antibodies were associated with more severe radiographic progression over 8 years in all included and ACPA-negative RA patients [45]. Similarly, Brink et al reported a more severe radiographic damage in $42.2 \%$ of RA patients positive for such antibodies over 2 years [46]. Anti-CarP antibodies have been also correlated with subclinical atherosclerosis and mortality in RA patients $[47,48]$. In the study by Spinelli et al, the association between anti-CarP antibodies and measures of endothelial dysfunction, used as markers of subclinical atherosclerosis, was described in 50 RA patients [47]. Finally, in a Spanish cohort of 331 RA patients, Vidal-Bralo et al. reported a correlation between anti-CarP antibodies and mortality in RA over a period of 9 years follow-up [48].

Despite providing a synthesis of available literature, our systematic review is impaired by different limitations, mainly due to lack of standardization of tests analysing anti-CarP antibodies and different RA characteristics across evaluated studies (i.e. different disease durations and applied classification criteria).

\subsection{The possible role of MBDA in RA management}

\subsubsection{In RA, the possible role of MBDA in predicting disease course and} response to treatments is still controversial. LoE 5

Multi-biomarker disease activity (MBDA) test has been developed evaluating serum levels of 12 proteins associated with RA disease activity $[49,50]$. In this systematic review of literature, we investigated the role of MBDA in RA, assessing its predictive role of radiographic progression and response to treatments. After screening titles and abstracts, 6 observational studies were retrieved and included in the review [51-56].

Conflicting results are published assessing the predictive value of MDBA for radiographic progression. In the study by Bakker et al., MBDA was not identified as predictor of radiographic progression over 2 years analysing 120 sera from CAMERA study, despite the association with disease activity [51]. Similarly, analysing sera and outcomes of 171 RA patients enrolled in the DRESS study, MBDA score was not predictive of radiographic progression, neither of successful tapering or subsequent flare, in patients who tapered TNFi [52]. In contrast, Hambardzumyan et al. reported MBDA score was an independent predictor of radiographic progression, analysing $235 \mathrm{RA}$ patients from SWEFOT trial, after 1 year of follow-up [53]. Fur- 
thermore, associations between MBDA score and imaging findings of radiographic damage, were investigated in $52 \mathrm{RA}$ patients enrolled in HURRAH trial, by using magnetic resonance imaging (MRI), ultrasonography (US), computed tomography and radiography. Despite the fact that the MBDA score poorly correlated with MRI/US indexes of inflammation, elevated values were determined in patients characterised by more severe radiographic damage [54].

Analysing MBDA and response to treatment, Hambardzumyan et al. described the MBDA to be predictive for optimal add-on treatment in non-responder RA patients [55]. Authors assessed data from 157 patients enrolled in SWEFOT trial, after 3 months of methotrexate (MTX) therapy. A significant percentage of patients characterised by a low MBDA score experienced a good clinical response to subsequent triple therapy (MTX + sulfasalazine + hydroxychloroquine), whereas in patients displaying a high MBDA score, a significant percentage experienced a good clinical response to subsequent combination therapy with TNFi (MTX + infliximab) [55]. Finally, in the analysis of RETRO study MBDA scores were investigated to be predictive of disease relapse. RETRO was a phase-3, multicentre, randomised, open, prospective, controlled, parallel-group study in patients were allocated to continue conventional and/or biological DMARD regimen at full dose for 12 months, to reduce the dose of all conventional and/or biological DMARDs by $50 \%$ or to reduce the dose of all conventional and/or biological DMARDs by $50 \%$ for the first 6 months before to entirely stop all DMARDs. The results showed that higher values of MBDA scores in patients experiencing disease relapse after tapering and/or stopping conventional and/or biological DMARDs treatment, allowing a prediction of relapse in more than $80 \%$ of the patients [56].

Future specific designed and adequately powered studies with a longer follow up are needed to fully clarify the role of MBDA in management of RA patients, in predicting radiographic progression as well as response to therapeutic strategies.

\section{Spondyloarthritides working group}

The spondyloarthritis (SpA) complex includes a group of inflammatory rheumatic diseases with peculiar clinical and radiological features including sacroiliitis, enthesitis, and dactylitis [57]. Among SpA, psoriatic arthritis (PsA) is characterized for a broad and heterogeneous spectrum of clinical features and courses [58,59]. In some cases, PsA can occur with peripheral enthesitis, particularly Achilles tendinitis, and/ or dactylitis $[60,61]$. In the last decades, an increasing attention has been paid to the erosive and deforming course of PsA (40\%-60\% of patients) [62]. The identification of clinical and biological predictors of response to different biological disease-modifying anti-rheumatic drugs (bDMARDs) might help clinicians to make evidence-based decisions that maximise the benefits from treatment by targeting subsets of patients. In addition, this approach could also improve the cost/benefit and benefit/risk ratios in patients selected to start bDMARDs treatment [63].

\subsection{Although enthesitis may be considered a clinical marker of PsA, there is no evidence showing that its presence at baseline predicts the response to different bDMARDs (TNFis, IL17is, IL12/IL23is). LoE 5}

Enthesitis is the inflammation of the insertion of tendons and ligaments into the bone and represents a hallmark of PsA and SpA [64]. It can be considered among the first signs of PsA, occurring independently of arthritis [60]. The role of enthesitis as a predictor of treatment response in ankylosing spondylitis (AS) has been well defined in 2011 by Vastesaeger et al. [65]. Thereafter, in 2013, in a retrospective study, Spadaro et al. and co-workers showed that in AS patients treated with adalimumab, etanercept or infliximab, the proba- bility of obtaining partial remission was significantly lower when enthesitis was present at baseline [66]. In PsA patients, there are no studies evaluating the role of enthesitis as an independent clinical biomarker predicting the response among different bDMARDs (TNFis, IL17is, IL12/ IL23is).

\subsection{Although data available on biomarkers for predicting therapeutic response in SpA are scarce, CRP in clinical practice may be useful in predicting TNFis response. LoE $2 b$}

Only few articles addressed the potential role of soluble biomarkers in prediction of treatment response to TNFis in SpA patients. The majority of these studies were characterised by a weak methodology, mainly due to low power or lack of power calculation and an uncontrolled design. Amongst the potential biomarkers, baseline serum levels of matrix metalloproteinase-3 (MMP-3) [67,68], serum type I collagen C-telopeptide (sCTX) [69], complement fraction C3 [70], serum amyloid A (SAA) [71], anti-drug antibodies (ADAbs) [72], and IL-6 [73] have been reported to predict treatment response in individual studies. Moreover, two well-designed sub-studies of RCTs evaluated the predictivity of a large pool of soluble biomarkers in AS and PsA [74,75]. In particular, baseline levels of insulin, apolipoprotein C3, leptin, haptoglobin, IL-6, osteocalcin, procollagen type 1 amino-terminal propeptide (P1NP) and deoxypyridinoline were associated with ASAS20 response at week 14 in AS patients while baseline levels of adiponectin, prostatic acid phosphatase (PAP) and vascular endothelial growth factor (VEGF) predicted at least two out of the three clinical endpoints (ACR20, DAS28, PASI75) after 14 weeks in PsA patients. The results of the systematic review showed that only higher baseline C-reactive protein CRP values were consistently reported to predict response to treatment with TNFis in SpA patients, although this evidence relies mainly on observational studies [71,74,76] and only one RCT [73].

\section{SSc working group}

SSc has the highest fatality rate among connective tissue diseases and is characterized by cellular and humoral immunological abnormalities, fibroproliferative vasculopathy, and fibrosis of the skin and various internal organs. Pulmonary involvement, including both interstitial lung disease (ILD) and pulmonary arterial hypertension (PAH) is currently the primary cause of morbidity and mortality in SSc [77]. The course of ILD is highly variable, and patients may develop severe and rapidly progressive interstitial lung involvement during the early phase of the disease, while others may have a limited and non-progressive course [78-81]. The clinical course of untreated PAH is in most cases rapidly progressive leading to respiratory failure or death within 2-3 years after it becomes clinically detectable [82]. In this context, the identification of biomarkers for assessing certain phenotypes associated with an increased risk of developing severe and rapidly progressive ILD and/ or PAH is extremely important [83]. At present, despite intense investigation, only a few biomarkers for SSc have been fully validated and widely accepted. On this background, five statements were formulated and voted.

\subsection{Anti-topoisomerase 1 antibody is a biomarker for faster progression of SSC-ILD. LoE $2 b$}

Anti-topoisomerase 1 antibody is directed against DNA topoisomerase I and is strongly associated with the diffuse form of SSc (dcSSc) and with the development and/or faster progression of ILD. The Genetics versus Environment in Scleroderma Outcome Study (GENISOS), based on a prospective, observational cohort of 266 patients with early systemic sclerosis, confirmed that the presence of 
anti-topoisomerase 1 antibody was the only variable associated with differential forced vital capacity (FVC) levels, predicting the rate of decline in FVC within the first 3 years of follow-up [80]. Subsequently, a study cohort consisting of 398 consecutive SSc patients with follow-up for up to 15 years confirmed that anti-topoisomerase 1 antibody remained remains a significant predictor of the development of clinically significant pulmonary fibrosis (PF) together with other variables such as dcSSc, lower FVC, and lower diffusing lung capacity for carbon monoxide (DLCO). In this study, SSc-ILD was confirmed by high-resolution computed tomography, and clinically significant SSc-ILD was defined as FVC or DLCO $\leq 55 \%$ predicted or a documented decline in FVC or DLCO of $\geq 15 \%$ [84]. However, there is no consensus for the definition of ILD progression.

\subsection{KL-6, SP-D, CCL18 may be considered as biomarkers for short-term progression of SSC-ILD. LoE $3 b$}

Krebs von den Lungen-6 (KL-6), surfactant protein-D (SP-D) and CC-chemokine ligand 18 (CCL18) are proteins produced and secreted by alveolar type II epithelial cells and alveolar macrophages, and were found to be increased in the serum of SSc patients compared to healthy subjects, and even further increased in SSc-ILD patients. Furthermore, these proteins appear to predict the development of SSc-ILD and subsequent FVC decline, in the short-term as well [85-94]. It is important to highlight that the definition of "short-term" ILD progression is herein defined as progression within 12 months, which reflects the duration of clinical trials in SSc-ILD.

CCL18 serum levels predicted SSc-ILD progression in one study [86]. These results were, subsequently, confirmed in a second independent cohort of SSc patients, with a different, lower cut- off value of CCL18 [87]. However, these differences in cut-off levels highlight the need of standardization of CCL18 assays, before its use as a biomarker in clinical practice [88]. Furthermore, increased serum KL-6 was identified as an independent predictor of subsequent FVC decline [89-91] and associated with ILD activity [92]. Finally, increased serum SP-D, was a significant predictor of FVC decline [92,94].

\subsection{The DETECT algorithm is useful to identify SSc patients at higher risk for PAH who should undergo RHC. LoE $2 a$}

In a large, multicentre, cross-sectional, study, the DETECT algorithm, a composite biomarker which uses clinical variables, pulmonary functional tests, immunological, biological, electrocardiographic and echocardiographic parameters, proved to be a useful tool, to identify patients who are more likely to have PAH, especially those who are asymptomatic. A positive score justifies performing right heart catheterization for confirmation of PAH [95]. Subsequent work, by different research groups in different cohorts of patients with SSc, confirmed the validity of this composite biomarker as an excellent screening method due to its high sensitivity and predictive negative value, minimizing missed diagnosis of PAH [96,97], also comparing it with European Society of Cardiology/European Respiratory Society (ESC/ERS 2009) guidelines [98]. Nonetheless, it should be kept in mind that the DETECT score was developed for SSc patients with low DLCO and disease duration $>3$ years and is therefore validated in this particular patient group.

\subsection{High levels of plasma CXCL4 may predict development of PAH in SSc patients. LoE $2 b$}

CXCL4 is a pro-inflammatory chemokine that regulates immune cells, such as T cells, monocytes, dendritic cells, as well as non-immune cells like endothelial cells, and may have an important role in inflammation and wound repair [99,100]. Recently, a proteome-wide analysis has demonstrated that CXCL4 is the predominant protein produced by SSc-derived plasmacytoid dendritic cells and it was identified as a potential biomarker associated with multiple organ involvement in SSc. Elevated plasma levels of CXCL4 strongly correlated with the extent of skin and lung fibrosis, as well as with early PAH development [101]. Despite the high LoE and GoR of the study, to date, no additional study has confirmed these data.

\subsection{High levels of serum Anti-AT1R and Anti-ETAR antibodies may predict development of PAH in SSc. LoE $2 b$}

Autoantibodies against the angiotensin II type 1 receptor (AT1R) and the endothelin-1 type A receptor (ATAR) have been shown to be elevated in the sera of most SSc patients, and associated with vascular and fibrotic SSc complications [102,103]. In particular, both anti-AT1R and anti-ETAR antibodies are predictive and prognostic markers of SSc-PAH [104].

\section{SLE working group}

Systemic Lupus Erythematosus (SLE) is a chronic autoimmune disease where treatment is usually long-term or even life-long. One of the most controversial aspects of the management of SLE lies in the need to define a treat-to-target strategy, developed under the influence of evidence, to tailor to individual patients [105]. Even if to date a generally accepted definition of remission is lacking, in recent years several studies have shown that remission is a pursued and reachable target in SLE that positively impact disease outcomes halting accrual of organ damage [106-108]. The disease management during remission is an outstanding unmet need in SLE and EULAR task force suggested that is not recommended to escalate the treatment in clinically asymptomatic patients solely on stable or persistent serological activity [105].

Despite significant advances in pathogenic knowledges and therapy, SLE is still burdened by significant morbidity and mortality. Epidemiological studies show that the goal of prolonged remission or low disease activity is achievable only in a part of SLE patients [106]. The majority of patients experience frequent disease flares, significant undesirable effects of treatments and irreversible organ damage accrual [109]. The development of new drugs and the use of available therapies in the framework of a treat-to-target approach might be essential to improve the long-term prognosis of the disease [105]. Belimumab, a monoclonal antibody directed against the B-cell activating factor (BAFF) [110], is the first drug approved for the treatment of SLE. Randomized clinical trials and real-life studies demonstrated the efficacy of Belimumab in reducing disease activity and flares, sparing glucocorticoids, improving fatigue and health related quality of life and in preventing damage accrual $[111,112]$. However, literature data and daily life clinical practice clearly demonstrated that only a part of SLE patients adequately respond to Belimumab [113]. The identification of drug response biomarkers could be crucial to optimize the use of Belimumab in SLE.

\subsection{There is no evidence that a negative serology predicts a successful withdrawal of therapy in SLE patients (with or without nephritis) in clinical remission. LOE 5}

Although the topic concerning treatment withdrawal is a priority aspect in the management of patients with SLE, the currently available data on this topic are still very fragmented. Reviewing the existing literature, it has not been possible to find studies that answer directly to our research questions reformulated according to the PICO methodology, nor to identify trials designed for evaluating treatment 
withdrawal in inactive SLE patients with negative serology. No data are available to suggest the optimal duration of treatment in responsive patients and a possible tapering strategy [114]. Threes studies, nevertheless, focused on SLE in complete remission (including inactive SLE with negative serology) without therapy (with or without antimalarials) $[106,108,115]$.

In the study by Urowitz et al., prolonged remission was defined as a 5 -year consecutive period of no disease activity (SLE disease activity index, SLEDAI $=0$ ) and without treatment (corticosteroids, antimalarials, or immunosuppressants). Out of 703 patients, only 12 patients (1.7\%) had prolonged complete remission of at least 5 years with no treatment [115]. Zen et al. defined remission as prolonged when lasting $\geq 5$ consecutive years. The complete remission corresponded to the absence of disease activity (SLEDAI-2000 $=0$ ) in corticosteroid-free and immunosuppressant-free patients. During the 5-year follow-up, 16 patients (7.1\%) achieved prolonged complete remission [106]. Medina-Quiñones et al. studied lupus patients achieving a complete remission, without clinical or serologic features and no treatment with steroids and immunosuppressive drugs for at least 3 years. Overall 77 patients $(14.5 \%)$ achieved complete remission for at least 3 years [108].

\subsection{In lupus nephritis, negative serology does not predict a successful withdrawal of therapy. LoE $3 b$}

Regarding lupus nephritis, only one study partially covered our research question. Moroni et al. reported their cumulative experience with treatment withdrawal in patients with biopsy-proven lupus nephritis with a follow-up of at least 5 years [116]. The authors completely stopped treatment in 52 of 161 (32\%) patients with class III, IV or V lupus nephritis. The decision of interrupting therapy was taken for those patients who had achieved a stable clinical remission and did not show any renal or extra-renal flare. Of 52 patients who stopped therapy, 32 $(61.5 \%)$ never developed new flares and continued without any therapy for the subsequent 101.8 months (range 44-180 months) of observation after interruption of treatment. The other 20 patients (38\%) had at least one flare. No difference in the prevalence of hypocomplementemia and anti-dsDNA antibodies was observed among patients with and without flares [116].

The therapeutic scheme to be adopted in lupus nephritis during complete remission, when and how to reduce corticosteroids and immunosuppressants are unanswered questions, as well as it is not clear the role of serology in spacing/tapering strategy [117]. Recently a position paper has specifically addressed this point [118]. The experts have emphasized that the essential prerequisite for a safe withdraw of therapy is patient's remission. The authors have remembered that special attention must be given to tapering slowly, progressively and under strict medical surveillance for avoiding severe or irreversible renal failure after an abrupt discontinuation [118].

\subsection{Available data demonstrated that baseline BAFF serum levels do not} predict response to Belimumab in SLE patients. Other baseline biomarkers, such as high dose of glucocorticoids, low complement fragment C3 or C4, positivity to anti-dsDNA, might be useful in predicting response to Belimumab. LoE $1 b$

Wallance et al. reported the results of the phase II randomized clinical trial on the use of Belimumab in addition to standard of care (SOC) in 449 active SLE patients [119]. Considering the serologically active subgroup (ANA titer $>1: 80$ and/or anti-dsDNA $>30 \mathrm{IU} / \mathrm{ml}$ ), a significant greater proportion of patients treated with Belimumab reached the SLE Responder Index (SRI) compared to placebo (46\% versus $29 \%$ ). In the exploratory subgroup analysis, the mean percent change in SELENA-SLEDAI from baseline to week 52 was compared after stratification of patients according several biomarkers. A significant reduction in SELENA-SLEDAI was observed in patients treated with daily dose of prednisone $\geq 7.5 \mathrm{mg}$, in seropositive patients, in patients positive for ANA or anti-dsDNA or with low complement fragment components C3 or C4. Nevertheless, the reduction of SELENA-SLEDAI in patients with serum BAFF above the limit of detection compared with patients with undetectable BAFF did not reach the statistical significance [119]. Roth et al. performed a post hoc analysis of pooled data from phase III clinical trials BLISS-52 and BLISS-76. In the efficacy analysis, 1108 SLE patients were stratified according to baseline BAFF serum levels ( $\geq 2 \mathrm{ng} / \mathrm{ml}$ verus $<2 \mathrm{ng} / \mathrm{ml}$ ). In both subgroups (high and low basal BAFF) about half of patients achieved the reduction of SELENA-SLEDAI of almost 4 points and the SRI response at week 52 [120]. Moreover, in both subgroups, the study outcomes were reached in the first 24 weeks and approximately maintained over time. The post-hoc analysis did not provide a direct statistical comparison of subgroups. However, numerically greater differences in study outcomes for Belimumab treated patients versus placebo were reported in the groups with high basal BAFF compared to the low basal BAFF group [120].

\section{APS working group}

Antiphospholipid syndrome (APS) is an autoimmune disease clinically characterized by vascular thrombosis and pregnancy morbidity. Diagnosis is confirmed by the persistent presence of anti-phospholipid antibodies (aPL) in patients' plasma [121]. At present, three aPL assays are included in the classification criteria: Lupus Anticoagulant (LA), anticardiolipin (aCL) antibodies and anti- $\beta 2$ glycoprotein I (anti- $\beta 2$ GPI) antibodies of IgG and/or IgM isotype [122]. In the last decade, a number of new tests, with variable sensibility and specificity, have been proposed as additional serological biomarkers of APS. Among them, anti-phosphatidylserine/prothrombin (anti-PS/PT) antibodies and epitope specific anti-beta2GPI antibodies have been suggested to display higher diagnostic and prognostic value for APS [123]. As a matter of fact, $\beta 2 \mathrm{GPI}$ and pro-thrombin have been addresses as the most important antigenic targets of aPL [123].

\subsection{Anti- $\beta 2$ GPI antibodies}

$\beta 2 \mathrm{GPI}$ is a positively charged glycoprotein composed by 5 domains. Several studies have shown that anti- $\beta 2$ GPI autoantibodies can be directed against different domains, even if domain I (D1) has been identified as the immune-dominant epitope in APS [124]. Moreover, this subpopulation of autoantibodies has been demonstrated to play a pathogenic role in APS [125-127]. On the contrary, antibodies targeting the fourth and fifth domain (D4/5) of the molecule have been identified in aPL asymptomatic carriers and in patients with infectious diseases, atopic dermatitis and babies born from patients with autoimmune diseases $[128,129]$. To assess the diagnostic and prognostic value of epitope specific anti-beta2GPI antibodies, a systematic review of the literature, from July 2007 to July 2017, including randomized clinical studies, observational studies and reviews and on APS, has been performed. Of the 25 potentially relevant publications, 15 studies have been selected for analysis. Pulling together the results of 11 papers, for a total of 1585 patients, the overall estimated median prevalence of anti-D1 antibodies was $43 \%$, ranging from $26.7 \%$ in SLE patients to $55.4 \%$ in primary APS [130].

\subsubsection{The addition of anti-domain I to laboratory classification criteria} seems to increase the risk of thrombosis. LoE5

Several different studies have shown a significant association of anti-D1 positivity to the risk of thrombosis, while the correlation 
with pregnancy morbidity was less evident [130-134]. Interestingly, anti-D1 positivity has been associated to triple aPL positivity, LA positivity and the presence of an additional autoimmune disease and higher anti-D1 titers have been observed in triple aPL positive APS patients and in patients with a history of thrombosis [130-134]. By contrast, only 3 studies reported the prevalence and clinical significance of anti-D4/5 autoantibodies. All these papers show that anti-D4/5 are more prevalent in asymptomatic aPL positive subjects $[128,129,135]$.

\subsubsection{There is no evidence that anti-domain I can substitute anti-beta2GPI I. LoE5}

Even if anti-D1 positivity has been clearly associated with the risk of thrombosis, a relevant prognostic value of this subgroup of autoantibodies for pregnancy complications is still to be confirmed [130-134]. In several different studies, anti-2GPI antibodies has been shown to display a higher prognostic value than anti-D1 for thrombotic events $[131,136,137]$. Moreover, Andreoli et al. have reported that a small but relevant proportion of APS patients can display anti-2GPI positivity even if they do not react with domain 1 [129]. At present, anti-domain I can be considered an additional, rather than an alternative, prognostic marker of APS.

\subsection{Anti-PS/PT antibodies}

Among new serological biomarkers of APS, antibodies specific to phospholipid-binding plasma proteins, phospholipid-protein complexes, and anionic phospholipids other than cardiolipin (including phosphatidylserine, phosphatidylinositol and phosphatidic acid). Antibodies against human prothrombin (aPT) and the complex of prothrombin bound phosphatidylserine (aPS/PT) have been strongly associated to the APS, even if their clinical relevance and diagnostic utility remain to be fully elucidated [138]. Although, it seems that aPT and aPS/PT belong to different autoantibodies families, they can both be present in the same patient [138-143].

\subsubsection{The presence of anti-PS/PT seems to increase the risk of clinical manifestations of APS. LoE5}

Current evidence suggest that aPS/PT measurement could help in the evaluation possible adverse pregnancy outcomes and thrombosis in patients suspected of suffering from APS and the assessment of thrombotic risk in patients with previous thrombosis and/or Systemic Lupus Erythematosus (SLE) [138,139]. A systematic review of Sciascia S, et al. reported an increased risk of thrombosis associated to aPT and aPS/ PT (OR 2.3 [95\%CI 1.72-3.5]) [139]. Moreover, aPS/PT seemed to be a stronger risk factor for thrombosis, both arterial and/or venous than aPT (OR 5.11 [95\%CI 4.2-6.3] and OR 1.82 [95\%CI 1.44-2.75], respectively). However, these promising associations are based on the results of 10 studies, almost all but one, retrospective reports [138]. Recently, the accuracy of IgG aPS/PT for APS diagnosis in heterogeneous population has been confirmed in a large multicenter study, reporting a higher prevalence of aPS/PT IgG in APS patients than in those without (47\% versus $12 \%$ ), with an OR of 6.4. In this study, sensitivity, specificity, LR + and LR- for APS diagnosis were 47\%, 88\%, 3.9 and 0.6, respectively [140]. Moreover, Hoxha et al. showed a positive association between IgM aPS/PT titres and both vascular thrombosis and pregnancy morbidity. Additionally, IgG aPS/PT was significantly associated with venous thrombosis [142].

\subsubsection{The presence of anti-PS/PT seems to identify very few patients with} the so called seronegative APS. LOE5

The concept of the so called seronegative APS (SNAPS) was introduced in 2003 to identify patients with highly suggestive manifesta- tions of APS, but persistently negative for the classification laboratory criteria [144]. In the past 5 years, research has been focused on the identification of "new" aPL, not included in the serological criteria, in seronegative APS patients $(3,20,22)$. Few studies have reported a relevant prevalence of anti-PS/PT antibodies in SNAPS patients. Amengual et al found an IgG aPS/PT antibody positivity in $6 \%$ of 17 patients with clinical manifestations of APS but negative for the classification laboratory criteria [141]. More recently, Shi et al, showed that $51 \%$ of patients with SNAPS had IgG and/or IgM anti-PS/PT antibodies and that the prevalence of anti-PS/PT was significantly higher in SNAPS patients compared to SLE patients [145].

Taking together these findings, anti-D1 and anti-PS/PT antibodies can currently be addressed as potential additional serological biomarkers of APS, that can help for a better stratification of thrombotic and obstetric risk in APS, in combination with the classification APS laboratory markers. More prospective clinical studies are needed in order to define the specific role of aPS/PT as a potential biomarker of APS diagnosis.

\section{8. pSS working group}

pSS is an autoimmune disease characterized by an inflammatory infiltrate affecting the exocrine glands, mainly the salivary and lacrimal glands, which may lead to a decrease in the glandular function. [146]. The main symptoms include dryness of the mouth and eyes. Some extra-glandular features may be observed, such as vasculitis, interstitial lung disease, interstitial nephritis or severe cryoglobulinaemic vasculitis and central nervous system involvement [147]. Furthermore, a significant percentage of pSS patients may develop B cell lymphoma [148]. Given the clinical heterogeneity and outcome of these patients, due to the different involvement observed in target organs, an early identification of the patient's subsets, particularly for the subgroup of patients prone to lymphoma development remains a major challenge [149]. So far, there is a strong need for new predictive clinical or biologic biomarkers, which could, at baseline, identify those patients with a poor outcome, and drive the best optimal therapeutic intervention, tailored to the clinical phenotype of the patients. Possible predictive biomarkers of unfavourable outcomes in pSS include serum CXCL13 and BAFF levels, and the presence of germinal centre (GC)-like structures in minor salivary gland (MSG) tissue. CXCL13 is a chemokine involved in the formation and maintenance of GC-like structures in autoimmune diseases [150]. Data suggest it may function as a biomarker of GC formation following vaccination for example [151]. BAFF is essential for B cell survival and GC maintenance [152,153]. Different studies reported higher levels of serum CXCL13 and BAFF in pSS patients who developed lymphoma [154,155]. GC-like structures in MSG biopsies are associated with more severe disease and with the presence of autoantibodies and their presence and function in MSG may be associated with the lymphoma risk [156]. At present, there is a general consensus in available literature that in pSS patients low C4, cryoglobulins, purpura, vasculitis and parotid swelling are strongly associated with lymphoma development [148].

\subsection{The predictive value of CXCL13 in pSS, for both poor prognosis and therapeutic response, remains to be clarified. LoE $2 b$}

CXCL13 is overexpressed in different chronic inflammatory diseases and is part of lymphoid tissue neogenesis, notably in the segregation of $\mathrm{T}$ and $\mathrm{B}$ cells into the T-cell zone and B-cell follicles [150]. In pSS salivary glands CXCL13 may directly control the formation and maintenance of functional ELSs and may promote, in a subset of patients the progressive development to lymphoma. CXCL13 levels significantly correlate with ESSDAI and, CXCL13 serum levels are 
significantly higher in pSS patients with an active disease (ESSDAI $\geq$ 5) $[150,154]$. In a Japanese cohort of 88 pSS patients CXCL13 serum levels correlate with lymphadenopathy, glandular, pulmonary and biologic domains. Furthermore, the Authors found a correlation between CXCL13 serum levels and hypergammaglobulinemia, underlying the possible correlation between the biomolecular status of salivary gland pathology and lymphomagenesis [158]. Furthermore, increased CXCL13 serum levels have been shown in pSS patients with the highest levels observed in patients with lymphoma [154]. Interestingly, the baseline CXCL13 levels of those patients who developed lymphoma during follow-up, were higher than the levels observed in patients who did not develop lymphoma [154]. These data should be confirmed in larger studies. In a small open label study of abatacept in pSS, reduction on CXCL13 levels correlated with reduction in ESSDAI scores [159], but this requires confirmation in larger, randomised controlled trials.

\subsection{The predictive value of baseline BAFF levels for lymphoma development and therapeutic response to RTX should be further assessed. LoE $2 b$}

BAFF levels are critical for peripheral B cell survival and differentiation, GC-like structure formation, plasma cell survival and IgG and IgE class switching. Due to these activities, BAFF may play a crucial role in pSS pathogenesis and in GC formation [160]. In fact, different studies show a correlation between BAFF serum levels and ESSDAI scores, especially for lymphadenopathy, glandular and pulmonary domains $[155,158]$. Furthermore, BAFF serum levels are higher in pSS patients with lymphoma and, in this setting, increased BAFF levels at diagnosis, are significantly associated with lymphoma development, as confirmed by multivariate analysis [154]. Increased BAFF serum levels also seem to be predictive of rituximab (RTX) treatment failure, suggesting a possible predictive role of BAFF serum levels in identifying a subset of patients suitable to be treated with this B-cell depleting agent [161]. On the contrary, data from a small open label study suggest that serum BAFF levels are not predictive for clinical to belimumab [162]. Although these results look very promising for personalized medicine, they have to be confirmed in larger cohorts before being helpful in daily clinical setting.

\subsection{The clinical utility of the presence of GC-like structures, in MSGs, to} predict lymphoma development should be further assessed with standardization of technique and multicentre studies given the relatively low incidence of lymphoma. LoE $3 b$

It has been shown that approximately $25 \%$ of pSS patients display GC-like structures in their salivary glands, and these patients have more severe disease in terms of higher salivary gland focus score, higher prevalence of rheumatoid factor, anti-SSA, and anti-SSB antibodies [163]. Approximately 5\% of patients with pSS will develop non-Hodgkin B-cell lymphomas of mucosal-associated lymphoid tissue, commonly in the parotid glands. Different reports describe the association between GC-like structures and lymphoma development, highlighting that the detection of GC-like structures, by H\&E staining, may be a highly predictive and an easy-to-obtain marker for lymphoma development [156]. Two different retrospective small cohort studies showed that the development of lymphoma in pSS patients was associated with the presence of GC-like structures in MSG biopsies at the time of diagnosis [163,164]. Another observational retrospective study did not find a strong association with lymphoma development but confirmed that GC-like structures in MSGs were associated with the presence of systemic manifestations [165]. The absence of GC-like structures in MSGs has been associated with a high negative predictive value for lymphoma [163]. However not all studies have confirmed an association of GC-like structures and lymphoma development $[166,167]$ and the majority of studies investigating this relationship have issues of study design, being generally retrospective studies, with small populations of patients, and using different methods of case identification.

The identification of the GC-like structures should ideally be confirmed by IHC in order to identify the degree of organisation. In fact, some IHC studies, have failed to confirm the presence of GC-like structures observed by H\&E [156]. Further work to standardise of markers to be used is required however due to the potential importance of this topic [168], a strong scientific agreement should be reached regarding the appropriate procedures to draw a definitive conclusion.

\section{Discussion}

This work derived from a systematic review of available literature and International Experts' Consensus may provide a comprehensive highlight of the role of biomarkers in management of patients affected by autoimmune rheumatic diseases treated by conventional and/or biological therapies. The main objective of this work would be to counsel physicians on the suitable way to address the possible role of mechanistic, clinical and therapeutic markers in this context. Our paper synthesises key points and new information, largely from recent or ongoing medical research derived from technical review, that may have implications for management of these patients [169].

The continued identification of new biomarkers specific to autoimmune rheumatic disease is crucial for translation into personalised medicine, in terms of patient management. In fact, the personalised medicine is an emerging practice of medicine that uses the patients' phenotype to guide decisions made in regard to the diagnosis, prevention and treatment of diseases [170]. Biomarkers profiling may be useful for tailoring the right therapeutic strategy for the right patient at the right time, and/or to onset of a specific complications [171,172].

Despite providing a comprehensive synthesis of the current available literature, this review is impaired from major limitations and, therefore, all the results should be cautiously interpreted. The main limitation is related to the poor methodological quality of a vast percentage of the included studies, mainly observational studies providing less reliable findings when compared with possible randomised controlled trials primarily and specifically designed. On these bases, it could be difficult to perform comparisons between extracted data and future specific designed studies are needed to entirely clarify these features. On the other side, it must be pointed out that autoimmune diseases are rare diseases and organising specific designed studies to investigate the role of biomarkers may be a challenge. In addition, our consensus statements do not provide specific algorithms or guidelines for practice because these depend on different variables, such as cost, expertise and practice circumstances.

In conclusion, the results derived from our systematic review and International Experts' Consensus confirmed that the better understanding and targeting of existing therapies is still an important field of research. Biomarkers and personalised medicine would represent the key points in the future management of patients affected by autoimmune rheumatic diseases.

\section{Competing interest}

None 


\section{Uncited reference}

\section{Acknowledgements}

The Authors thank Mrs Federica Sensini for her technical assistance

\section{References}

[1] F. Goldblatt, S.G. O'Neill, Clinical aspects of autoimmune rheumatic diseases, Lancet 382 (9894) (2013) 797-808.

[2] M. Wahren-Herlenius, T. Dörner, Immunopathogenic mechanisms of systemic autoimmune disease, Lancet 382 (9894) (2013) 819-831.

[3] M.G.P. Zuidgeest, P.M.J. Welsing, G.J.M.W. van Thiel, A. Ciaglia, R. Alfonso-Cristancho, L. Eckert, et al., Egger M; WP3 of the GetReal consortium. Series: Pragmatic trials and real world evidence: Paper 5. Usual care and real life comparators, J Clin Epidemiol 90 (2017 Oct) 92-98.

[4] M.G.P. Zuidgeest, I. Goetz, R.H.H. Groenwold, E. Irving, G.J.M.W. van Thiel, D.E Grobbee, et al., Series: Pragmatic trials and real world evidence: Paper 1. Introduction, J Clin Epidemiol 88 (2017 Aug) 7-13.

[5] C. Mohan, S. Assassi, Biomarkers in rheumatic diseases: how can they facilitate diagnosis and assessment of disease activity?, BMJ 351 (2015 Nov 26) h5079.

[6] A. Consolaro, G.C. Varnier, A. Martini, A. Ravelli, Advances in biomarkers for paediatric rheumatic diseases, Nat Rev Rheumatol 11 (5) (2015 May) 265-275.

[7] L.J. Lesko, A.J. Jr Atkinson, Use of biomarkers and surrogate endpoints in drug development and regulatory decision making: criteria, validation, strategies, Annu Rev Pharmacol Toxicol 41 (2001) 347-366.

[8] M. Mayr, J. Zhang, A.S. Greene, D. Gutterman, J. Perloff, P. Ping, Proteomics-based development of biomarkers in cardiovascular disease: mechanistic, clinical, and therapeutic insights, Mol Cell Proteomics 5 (10) (2006 Oct) 1853-1864.

[9] W.H. Robinson, T.M. Lindstrom, R.K. Cheung, J. Sokolove, Mechanistic biomarkers for clinical decision making in rheumatic diseases, Nat Rev Rheumatol 9 (5) (2013 May) 267-276.

[10] T. Wu, C. Mohan, Proteomics on the diagnostic horizon: Lessons from rheumatology, Am J Med Sci 333 (1) (2007 Jan) 16-25.

[11] W.H. Robinson, R. Mao, Biomarkers to guide clinical therapeutics in rheumatology?, Curr Opin Rheumatol 28 (2) (2016 Mar) 168-175.

[12] R. Giacomelli, A. Afeltra, A. Alunno, C. Baldini, E. Bartoloni-Bocci, O. Berardicurti, et al., International consensus: What else can we do to improve diagnosis and therapeutic strategies in patients affected by autoimmune rheumatic disease (rheumatoid arthritis, spondyloarthritides, systemic sclerosis, systemic lupus erythematosus, antiphospholipid syndrome and Sjogren's syndrome)?: The unmet needs and the clinical grey zone in autoimmune disease management, Autoim mun Rev 16 (9) (2017 Sep) 911-924.

[13] M.M. Nielen, D. van Schaardenburg, H.W. Reesink, R.J. van de Stadt, I.E. van der Horst-Bruinsma, M.H. de Koning, et al., Specific autoantibodies precede the symptoms of rheumatoid arthritis: a study of serial measurements in blood donors, Arthritis Rheum 50 (2) (2004 Feb) 380-386.

[14] Aletaha D, Neogi T, Silman AJ, Funovits J, Felson DT, Bingham CO 3rd, Birnbaum NS, Burmester GR, Bykerk VP, Cohen MD, Combe B, Costenbader KH, Dougados M, Emery P, Ferraccioli G, Hazes JM, Hobbs K, Huizinga TW, Kavanaugh A, Kay J, Kvien TK, Laing T, Mease P, Ménard HA, Moreland LW, Naden RL, Pincus T, Smolen JS, Stanislawska-Biernat E, Symmons D, Tak PP, Upchurch KS, Vencovsky J, Wolfe F, Hawker G. 2010 rheumatoid arthritis classification criteria: an American College of Rheumatology/European League Against Rheumatism collaborative initiative. Ann Rheum Dis 2010 Sep;69(9):1580-8.

15] M.K. Demoruelle, M.C. Parish, L.A. Derber, J.R. Kolfenbach, J.M. Hughes-Austin, M.H. Weisman, et al., Performance of anti-cyclic citrullinated Peptide assays differs in subjects at increased risk of rheumatoid arthritis and subjects with established disease, Arthritis Rheum 65 (9) (2013 Sep) 2243-2252.

[16] A.H. Hensvold, T. Frisell, P.K. Magnusson, R. Holmdahl, J. Askling, A.I. Catrina, How well do ACPA discriminate and predict RA in the general population: a study based on 12590 population-representative Swedish twins, Ann Rheum Dis 76 (1) (2017 Jan) 119-125.

[17] S. Abolghasemi, et al., The sensitivity, specificity and accuracy of anti-citrulline antibody test in diagnosis of rheumatoid arthritis, Rheumatol Int 33 (4) (2013 Apr) 1027-1030.

[18] J. Payet, et al., Anticyclic citrullinated peptide antibodies in rheumatoid and nonrheumatoid rheumatic disorders: experience with 1162 patients, J Rheumatol 41 (12) (2014 Dec) 2395-2402

[19] B. Heidari, et al., The prevalence and diagnostic performance of anti-cyclic citrul linated peptide antibody in rheumatoid arthritis: the predictive and discriminative ability of serum antibody level in recognizing rheumatoid arthritis, Ann Saudi Med 29 (6) (2009 Nov-Dec) 467-470.

[20] H. Li, et al., Diagnostic value of anti-cyclic citrullinated peptide antibodies in northern Chinese Han patients with rheumatoid arthritis and its correlation with disease activity, Clin Rheumatol 29 (4) (2010 Apr) 413-417.
[21] D.A. Kim, et al., Is serum anti-cyclic citrullinated peptide level useful in the diagnosis of rheumatoid arthritis?, Clin Chim Acta 413 (7-8) (2012 Apr 11) 831-832.

[22] D. Pietrapertosa, et al., Diagnostic performance of anti-citrullinated peptide antibodies for the diagnosis of rheumatoid arthritis: the relevance of likelihood ratios, Clin Chem Lab Med 48 (6) (2010 Jun) 829-834.

[23] S.W. Syversen, et al., High anti-cyclic citrullinated peptide levels and an algorithm of four variables predict radiographic progression in patients with rheumatoid arthritis: results from a 10-year longitudinal study, Ann Rheum Dis 67 (2) (2008 Feb) 212-217.

[24] N. Vastesaeger, et al., A pilot risk model for the prediction of rapid radiographic progression in rheumatoid arthritis, Rheumatology (Oxford) 48 (9) (2009 Sep) 1114-1121, https://doi.org/10.1093/rheumatology/kep155.

[25] M.P. van der Linden, et al., Van Der Linden MPM, et al. Toward a data-driven evaluation of the 2010 American College of Rheumatology/European League Against Rheumatism Criteria for rheumatoid arthritis. Is it sensible to look at levels of rheumatoid factor?, Arthritis Rheum 63 (5) (2011) 1190-1199.

[26] C. Hecht, et al., Additive effect of anti-citrullinated protein antibodies and rheumatoid factor on bone erosions in patients with RA, Ann Rheum Dis 74 (12) (2015 Dec) 2151-2156.

[27] K. Shiozawa, et al., Anticitrullinated protein antibody, but not its titer, is a predictor of radiographic progression and disease activity in rheumatoid arthritis, $J$ Rheumatol 39 (4) (2012 Apr) 694-700.

[28] D. Aletaha, et al., Rheumatoid factor determines structural progression of rheumatoid arthritis dependent and independent of disease activity, Ann Rheum Dis 72 (6) (2013 Jun) 875-880.

[29] A.S. Avdeeva, et al., The relationship of antibodies to modified citrullinated vimentin and markers of bone and cartilage destruction in rheumatoid arthritis, Int J Rheumatol 2014 (2014), 464585.

[30] S.W. Syversen, et al., Prediction of radiographic progression in rheumatoid arthritis and the role of antibodies against mutated citrullinated vimentin: results from a 10-year prospective study, Ann Rheum Dis 69 (2) (2010 Feb) 345-351.

[31] T. Koga, et al., Anti-citrullinated peptide antibodies are the strongest predictor of clinically relevant radiographic progression in rheumatoid arthritis patients achieving remission or low disease activity: A post hoc analysis of a nationwide cohort in Japan, PLoS One 12 (5) (2017).

[32] B.J. Miriovsky, et al., Anti-CCP antibody and rheumatoid factor concentrations predict greater disease activity in men with rheumatoid arthritis, Ann Rheum Dis 69 (7) (2010 Jul) 1292-1297.

[33] C. Pomirleanu, et al., A predictive model for remission and low disease activity in patients with established rheumatoid arthritis receiving TNF blockers, Clin Rheumatol 32 (5) (2013 May) 665-670.

[34] E. Salgado, et al., Rheumatoid factor and response to TNF antagonists in rheumatoid arthritis: systematic review and meta-analysis of observational studies, Joint Bone Spine 81 (1) (2014 Jan) 41-50.

[35] R. Klaasen, et al., The value of rheumatoid factor and anti-citrullinated protein antibodies as predictors of response to infliximab in rheumatoid arthritis: an exploratory study, Rheumatology (Oxford) 50 (8) (2011 Aug) 1487-1493.

[36] E. Salgado, et al., Rheumatoid factor does not predict response to TNF antagonists in rheumatoid arthritis: three centers experience, Joint Bone Spine 80 (4) (2013 Jul) 438-440.

[37] P. Lal, et al., Inflammation and autoantibody markers identify rheumatoid arthritis patients with enhanced clinical benefit following rituximab treatment, Arthritis Rheum 63 (12) (2011 Dec) 3681-3691.

[38] M. Cuchacovich, et al., Basal anti-cyclic citrullinated peptide (anti-CCP) antibody levels and a decrease in anti-CCP titersare associated with clinical response to adalimumab in rheumatoid arthritis, Clin Exp Rheumatol 26 (6) (2008 Nov-Dec) 1067-1073.

[39] J. Narvaez, et al., Predictors of response to rituximab in patients with active rheumatoid arthritis and inadequate response to anti-TNF agents or traditional DMARDs, Clin Exp Rheumatol 29 (6) (2011 Nov-Dec) 991-997, Epub 2011 Dec 22.

[40] J. Sokolove, et al., Impact of baseline anti-cyclic citrullinated peptide-2 antibody concentration on efficacy outcomes following treatment with subcutaneous abatacept or adalimumab: 2-year results from the AMPLE trial, Ann Rheum Dis 75 (4) (2016) 709-714.

[41] G.J. Pruijn, Citrullination and carbamylation in the pathophysiology of rheumatoid arthritis, Front Immunol 6 (2015 Apr 27) 192

[42] M.K. Verheul, P.A. van Veelen, M.A.M. van Delft, A. de Ru, G.M.C. Janssen, T. Rispens, et al., Pitfalls in the detection of citrullination and carbamylation, Autoimmun Rev 17 (2) (2018 Feb) 136-141.

[43] L. Li, C. Deng, S. Chen, S. Zhang, Z. Wu, C. Hu, et al., Meta-Analysis: Diagnostic Accuracy of Anti-Carbamylated Protein Antibody for Rheumatoid Arthritis, PLoS One 11 (7) (2016 Jul 20), e0159000.

[44] J. Shi, R. Knevel, P. Suwannalai, M.P. van der Linden, G.M. Janssen, P.A. van Veelen, et al., Autoantibodies recognizing carbamylated proteins are present in sera of patients with rheumatoid arthritis and predict joint damage, Proc Natl Acad Sci U S A 108 (42) (2011 Oct 18) 17372-17377.

[45] S. Ajeganova, H.W. van Steenbergen, M.K. Verheul, K. Forslind, I. Hafström, R.E. Toes, et al., The association between anti-carbamylated protein (anti-CarP) antibodies and radiographic progression in early rheumatoid arthritis: a study exploring replication and the added value to ACPA and rheumatoid factor, Ann Rheum Dis 76 (1) (2017 Jan) 112-118.

[46] M. Brink, M.K. Verheul, J. Rönnelid, E. Berglin, R. Holmdahl, R.E. Toes, et al., Anti-carbamylated protein antibodies in the pre-symptomatic phase of rheumatoid arthritis, their relationship with multiple anti-citrulline peptide 
antibodies and association with radiological damage, Arthritis Res Ther 17 (2015 Feb 7) 25.

[47] F.R. Spinelli, A. Pecani, F. Ciciarello, T. Colasanti, M. Di Franco, F. Miranda, et al., Association between antibodies to carbamylated proteins and subclinical atherosclerosis in rheumatoid arthritis patients, BMC Musculoskelet Disord 18 (1) (2017 May 25) 214.

[48] L. Vidal-Bralo, E. Perez-Pampin, C. Regueiro, A. Montes, R. Varela, M.D. Boveda, Gomez-Reino JJ Gonzalez A. Anti-carbamylated protein autoantibodies associated with mortality in Spanish rheumatoid arthritis patients, PLoS One 12 (7) (2017 Jul 3), e0180144.

[49] J.W. Peabody, V. Strand, R. Shimkhada, R. Lee, D. Chernoff, Impact of rheumatoid arthritis disease activity test on clinical practice, PLoS One 8 (5) (2013 May 7), e63215.

[50] R. Fleischmann, S.E. Connolly, M.A. Maldonado, M. Schiff, Brief Report: Estimat ing Disease Activity Using Multi-Biomarker Disease Activity Scores in Rheumatoid Arthritis Patients Treated With Abatacept or Adalimumab, Arthritis Rheum 68 (9) (2016 Sep) 2083-2089.

[51] M.F. Bakker, G. Cavet, J.W. Jacobs, J.W. Bijlsma, D.J. Haney, Y. Shen, et al., Per formance of a multi-biomarker score measuring rheumatoid arthritis disease activity in the CAMERA tight control study, Ann Rheum Dis 71 (10) (2012 Oct) 1692-1697.

[52] C.A.M. Bouman, A. van der Maas, N. van Herwaarden, E.H. Sasso, F.H.J. van den Hoogen, A.A. den Broeder, A multi-biomarker score measuring disease activity in rheumatoid arthritis patients tapering adalimumab or etanercept: predictive value for clinical and radiographic outcomes, Rheumatology (Oxford) 56 (6) (2017 Jun 1) 973-980.

[53] K. Hambardzumyan, R. Bolce, S. Saevarsdottir, S.E. Cruickshank, E.H. Sasso, D Chernoff, et al., Pretreatment multi-biomarker disease activity score and radiographic progression in early RA: results from the SWEFOT trial, Ann Rheum Dis 74 (6) (2015 Jun) 1102-1109.

[54] S. Krabbe, R. Bolce, C.H. Brahe, U.M. Døhn, B.J. Ejbjerg, M.L. Hetland, et al., Investigation of a multi-biomarker disease activity score in rheumatoid arthritis by comparison with magnetic resonance imaging, computed tomography, ultrasonography, and radiography parameters of inflammation and damage, Scand J Rheumatol 46 (5) (2017 Sep) 353-358.

[55] K. Hambardzumyan, S. Saevarsdottir, K. Forslind, I.F. Petersson, J.K. Wallman, S Ernestam, et al., A multi-biomarker disease activity score and the choice of second-line therapy in early rheumatoid arthritis after methotrexate failure, Arthritis Rheum 69 (5) (2017 May) 953-963.

[56] J. Rech, A.J. Hueber, S. Finzel, M. Englbrecht, J. Haschka, B. Manger, et al., Prediction of disease relapses by multibiomarker disease activity and autoantibody status in patients with rheumatoid arthritis on tapering DMARD treatment, Ann Rheum Dis 75 (9) (2016 Sep) 1637-1644.

[57] J. Sieper, D. Poddubnyy, Axial spondyloarthritis, Lancet 390 (10089) (2017) 73-84.

[58] M.S. Chimenti, E. Ballanti, C. Perricone, P. Cipriani, R. Giacomelli, R. Perricone, Immunomodulation in Psoriatic arthritis: Focus on cellular and molecular pathways, Autoimmun Rev 12 (5) (2013) 599-606.

[59] R. Scarpa, F. Caso, L. Costa, R. Peluso, A. Del Puente, I. Olivieri, Psoriatic Disease 10 Years Later, J Rheumatol 44 (9) (2017 Sep) 1298-1301.

[60] C. Salvarani, F. Cantini, I. Olivieri, P. Macchioni, L. Niccoli, A. Padula, et al., Isolated peripheral enthesitis and/or dactylitis: a subset of psoriatic arthritis, J Rheumatol 24 (6) (1997 Jun) 1106-1110.

61] F. Caso, L. Costa, M. Atteno, A. Del Puente, L. Cantarini, E. Lubrano, et al., Simple clinical indicators for early psoriatic arthritis detection, Springerplus 3 (2014) 759.

62] S. D'Angelo, G. Tramontano, M. Gilio, P. Leccese, I. Olivieri, Review of the treatment of psoriatic arthritis with biological agents: choice of drug for initial therapy and switch therapy for non-responders, Open Access Rheumatol 9 (2017 Mar 2) 21-28.

[63] P.D. Carvalho, C. Duarte, E. Vieira-Sousa, L. Cunha-Miranda, P. Avila-Ribeiro, H Santos, et al., Predictors of response to TNF blockers in patients with polyarticular psoriatic arthritis, Acta Reumatol Port 42 (1) (2017 Jan-Mar) 55-65.

[64] G. Schett, R.J. Lories, M.A. D'Agostino, D. Elewaut, B. Kirkham, E.R. Soriano, et al., Enthesitis: from pathophysiology to treatment, Nat Rev Rheumatol 13 (12) (2017 Nov 21) 731-741.

[65] N. Vastesaeger, D. van der Heijde, R.D. Inman, et al., Predicting the outcome of ankylosing spondylitis therapy, Ann Rheum Dis 70 (2011) 97381.

[66] A. Spadaro, E. Lubrano, A. Marchesoni, S. D'Angelo, R. Ramonda, O. Addimanda, et al., Remission in ankylosing spondylitis treated with anti-TNF- $\alpha$ drugs: a national multicentre study, Rheumatology (Oxford) 52 (10) (2013 Oct) 1914-1919.

[67] S. Arends, E. van der Veer, H. Groen, P.M. Houtman, T.L. Jansen, M.K. Leijsma, et al., Serum MMP-3 level as a biomarker for monitoring and predicting response to etanercept treatment in ankylosing spondylitis, J Rheumatol 38 (8) (2011 Aug) 1644-1650.

[68] V. Chandran, H. Shen, R.A. Pollock, F.J. Pellett, A. Carty, R.J. Cook, et al., Soluble biomarkers associated with response to treatment with tumor necrosis factor inhibitors in psoriatic arthritis, J Rheumatol 40 (6) (2013 Jun) 866-871.

[69] S. Arends, A. Spoorenberg, P.M. Houtman, M.K. Leijsma, R. Bos, C.G. Kallenberg, et al., The effect of three years of TNFo blocking therapy on markers of bone turnover and their predictive value for treatment discontinuation in patients with ankylosing spondylitis: a prospective longitudinal observational cohort study, Arthritis Res Ther 14 (2) (2012 Apr 30) R98.

[70] M.S. Chimenti, C. Perricone, D. Graceffa, G. Di Muzio, E. Ballanti, M.D. Guarino, et al., Complement system in psoriatic arthritis: a useful marker in response prediction and monitoring of anti-TNF treatment, Clin Exp Rheumatol 30 (1) (2012 Jan-Feb) 23-30.
[71] M.K. de Vries, I.C. van Eijk, I.E. van der Horst-Bruinsma, M.J. Peters, M.T. Nurmohamed, B.A. Dijkmans, et al., Erythrocyte sedimentation rate, C-reactive protein level, and serum amyloid a protein for patient selection and monitoring of anti-tumor necrosis factor treatment in ankylosing spondylitis, Arthritis Rheum 61 (11) (2009 Nov 15) 1484-1490.

[72] J. Hwang, H.M. Kim, H. Jeong, J. Lee, J.K. Ahn, E.M. Koh, et al., Higher body mass index and anti-drug antibodies predict the discontinuation of anti-TNF agents in Korean patients with axial spondyloarthritis, Rev Bras Reumatol 2016 57 (4) (2017) 311-319, Jul - Aug.

[73] S. Visvanathan, C. Wagner, J.C. Marini, D. Baker, T. Gathany, J. Han, et al., Inflammatory biomarkers, disease activity and spinal disease measures in patients with ankylosing spondylitis after treatment with infliximab, Ann Rheum Dis 67 (4) (2008 Apr) 511-517.

[74] J. Gratacós, E. Casado, J. Real, J.C. Torre-Alonso, Prediction of major clinical response (ACR50) to infliximab in psoriatic arthritis refractory to methotrexate, Ann Rheum Dis 66 (4) (2007 Apr) 493-497.

[75] C. Wagner, S. Visvanathan, J. Braun, D. van der Heijde, A. Deodhar, B. Hsu, et al., Serum markers associated with clinical improvement in patients with ankylosing spondylitis treated with golimumab, Ann Rheum Dis 71 (5) (2012 May) 674-680.

[76] S. Iervolino, M.N. Di Minno, R. Peluso, M. Lofrano, A. Russolillo, G. Di Minno, et al., Predictors of early minimal disease activity in patients with psoriatic arthritis treated with tumor necrosis factor- $\alpha$ blockers, J Rheumatol 39 (3) (2012 Mar) 568-573.

[77] V.D. Steen, T.A. Medsger, Changes in causes of death in systemic sclerosis, 1972-2002, Ann Rheum Dis 66 (7) (2007 Jul) 940-944, (Epub2007 Feb 28).

[78] V.D. Steen, C. Conte, G.R. Owens, T.A. Medsger Jr, Severe restrictive lung disease in systemic sclerosis, Arthritis Rheum 37 (1994) 1283-1289.

[79] V.D. Steen, T.A. Medsger Jr, Severe organ involvement in systemic sclerosis with diffuse scleroderma, Arthritis Rheum 43 (2000) 2437-2444.

[80] S. Assassi, R. Sharif, R.E. Lasky, T.A. McNearney, R.M. Estrada-Y-Martin, H. Draeger, et al., Predictors of interstitial lung disease in early systemic sclerosis: a prospective longitudinal study of the GENISOS cohort, Arthritis Res Ther 12 (5) (2010) R166.

[81] D. Khanna, C.H. Tseng, N. Farmani, V. Steen, D.E. Furst, P.J. Clements, et al., Clinical course of lung physiology in patients with scleroderma and interstitia lung disease: analysis of the Scleroderma Lung Study Placebo Group, Arthritis Rheum 63 (2011) 3078-3085.

[82] V. Castro Susan, S.A. Jimenez, Biomarkers in systemic sclerosis, Biomark Med 4 (1) (2010 Feb) 133-147.

[83] M. Iudici, G. Moroncini, P. Cipriani, R. Giacomelli, A. Gabrielli, G. Valentini, Where are we going in the management of interstitial lung disease in patients with systemic sclerosis?, Autoimmun Rev 14 (7) (2015 Jul) 575-578, (ISSN: 15689972)

[84] S.I. Nihtyanova, B.E. Schreiber, V.H. Ong, D. Rosenberg, P. Moinzadeh, J.G. Coghlan, et al., Prediction of pulmonary complications and long-term survival in systemic sclerosis, Arthritis Rheum 66 (6) (2014 Jun) 1625-1635.

[85] M. Elhaj, J. Charles, C. Pedroza, X. Liu, X. Zhou, R.M. Estrada-Y-Martin, et al., Can serum surfactant protein D or CC-chemokine ligand 18 predict outcome of interstitial lung disease in patients with early systemic sclerosis?, J Rheumatol 40 (7) (2013 Jul) 1114-1120.

[86] K.P. Tiev, T. Hua-Huy, A. Kettaneh, M. Gain, S. Duong-Quy, C. Tolédano, et al., Serum CC chemokine ligand-18 predicts lung disease worsening in systemic sclerosis, EurRespir J 38 (6) (2011 Dec) 1355-1360.

[87] J. Schupp, M. Becker, J. Günther, J. Müller-Quernheim, G. Riemekasten, A. Prasse, Serum CCL18 is predictive for lung disease progression and mortality in systemic sclerosis, EurRespir J 43 (2014) 1530-1532.

[88] A.M. Hoffmann-Vold, A.H. Tennøe, T. Garen, $\emptyset$. Midtvedt, A. Abraityte, T.M Aaløkken, et al., High Level of Chemokine CCL18 Is Associated With Pulmonary Function Deterioration, Lung Fibrosis Progression, and Reduced Survival in Systemic Sclerosis, Chest 150 (2) (2016 Aug) 299-306.

[89] M. Kuwana, Y. Shirai, T. Takeuchi, Elevated Serum Krebs von den Lungen-6 in Early Disease Predicts Subsequent Deterioration of Pulmonary Function in Patients with Systemic Sclerosis and Interstitial Lung Disease, J Rheumatol 43 (10) (2016 Oct) 1825-1831.

[90] T.A. Winstone, D. Assayag, P.G. Wilcox, J.V. Dunne, C.J. Hague, J. Leipsic, et al., Predictors of mortality and progression in scleroderma-associated interstitial lung disease: a systematic review, Chest 146 (2) (2014 Aug) 422-436.

[91] K. Yanaba, M. Hasegawa, K. Takehara, S. Sato, Comparative study of serum surfactant protein-D and KL-6 concentrations in patients with systemic sclerosis as markers for monitoring the activity of pulmonary fibrosis, $\mathrm{J}$ Rheumatol 31 (2004) 1112-1120.

[92] Yamakawa H, Hagiwara E, Kitamura H, Yamanaka Y, Ikeda S, Sekine A, Baba T, Okudela K, Iwasawa T, Takemura T, Kuwano K, Ogura. Serum KL-6 and surfactant protein-D as monitoring and predictive markers of interstitial lung disease in patients with systemic sclerosis and mixed connective tissue disease. T J Thorac Dis 2017 Feb;9(2):362-371

[93] B. Kennedy, P. Branagan, F. Moloney, M. Haroon, O.J. O'Connell, T.M. O'Connor, et al., Sarcoidosis Biomarkers to identify ILD and predict lung function decline in scleroderma lung disease or idiopathic pulmonary fibrosis, Vasc Diffuse Lung Dis 32 (3) (2015 Sep 14) 228-236.

[94] G. Kumánovics, T. Minier, J. Radics, L. Pálinkás, T. Berki, L. Czirják, Comprehensive investigation of novel serum markers of pulmonary fibrosis associated with systemic sclerosis and dermato/polymyositis, ClinExpRheumatol 26 (3) (2008 May-Jun) 414-420.

[95] J.G. Coghlan, C.P. Denton, E. Grünig, D. Bonderman, O. Distler, D. Khanna, et al., Seibold JR; DETECT study group. Evidence-based detection of pulmonary arterial 
[96] T. Soukup, R. Pudil, K. Kubinova, L. Hromadkova, J. Dusek, M. Tosovsky, et al., Application of the DETECT algorithm for detection of risk of pulmonary arterial hypertension in systemic sclerosis: data from a Czech tertiary centre, Rheumatology (Oxford) 55 (1) (2016 Jan) 109-114.

[97] Y. Hao, V. Thakkar, W. Stevens, K. Morrisroe, D. Prior, C. Rabusa, et al., A comparison of the predictive accuracy of three screening models for pulmonary arterial hypertension in systemic sclerosis, Arthritis Res Ther 17 (2015 Jan 18) 7.

[98] Guillén-Del Castillo A1, Callejas-Moraga EL2, García G3, Rodríguez-Palomares JF3, Román A4, Berastegui C4, et al., Simeón-Aznar CP2. High sensitivity and negative predictive value of the DETECT algorithm for an early diagnosis of pulmonary arterial hypertension in systemic sclerosis: application in a single center, Arthritis Res Ther 19 (1) (2017 Jun 14) 135.

[99] A.J. Affandi, T.R. Radstake, W. Marut, Update on biomarkers in systemic sclerosis: tools for diagnosis and treatment, SeminImmunopathol 37 (5) (2015 Sep) 475-487.

[100] M. Hasegawa, Biomarkers in systemic sclerosis: Their potential to predict clinical courses, J Dermatol 43 (1) (2016 Jan) 29-38.

[101] L. van Bon, A.J. Affandi, J. Broen, R.B. Christmann, R.J. Marijnissen, L. Stawski, et al., Proteome-wide analysis and CXCL4 as a biomarker in systemic sclerosis, $\mathrm{N}$ Engl J Med 370 (5) (2014 Jan 30) 433-443.

[102] G. Riemekasten, A. Philippe, M. Näther, T. Slowinski, D.N. Müller, H. Heidecke, et al., Involvement of functional autoantibodies against vascular receptors in systemic sclerosis, Ann Rheum Dis 70 (3) (2011 Mar) 530-536.

[103] M.O. Becker, A. Kill, M. Kutsche, J. Guenther, A. Rose, C. Tabeling, et al., Vascular receptor autoantibodies in pulmonary arterial hypertension associated with systemic sclerosis, Am J RespirCrit Care Med 190 (7) (2014 Oct 1) 808-817.

[104] M.O. Becker, A. Kill, M. Kutsche, J. Guenther, A. Rose, C. Tabeling, et al., Vascular receptor autoantibodies in pulmonary arterial hypertension associated with systemic sclerosis, Am J Respir Crit Care Med 190 (7) (2014 Oct 1) 808-817.

[105] R.F. van Vollenhoven, M. Mosca, G. Bertsias, D. Isenberg, A. Kuhn, K. Lerstrøm, et al., Treat-to-target in systemic lupus erythematosus: recommendations from an international task force, Ann Rheum Dis 73 (2014) 958-967, https://doi.org/10. 1136/annrheumdis-2013-205139.

[106] M. Zen, L. Iaccarino, M. Gatto, S. Bettio, L. Nalotto, A. Ghirardello, et al., Prolonged remission in Caucasian patients with SLE: prevalence and outcomes, Ann Rheum Dis 74 (2015) 2117-2122, https://doi.org/10.1136/ annrheumdis-2015-207347.

[107] M. Zen, L. Iaccarino, M. Gatto, S. Bettio, F. Saccon, A. Ghirardello, et al., The effect of different durations of remission on damage accrual: results from a prospective monocentric cohort of Caucasian patients, Ann Rheum Dis 76 (2017) 562-565, https://doi.org/10.1136/annrheumdis-2016-210154.

[108] C.V. Medina-Quiñones, L. Ramos-Merino, P. Ruiz-Sada, D. Isenberg, Analysis of Complete Remission in Systemic Lupus Erythematosus Patients Over a 32-Year Period: SLE Remission Analysis, Arthritis Care Res 68 (2016) 981-987, https:// doi.org/10.1002/acr.22774

[109] T. Stoll, N. Sutcliffe, J. Mach, R. Klaghofer, D.A. Isenberg, Analysis of the relationship between disease activity and damage in patients with systemic lupus erythematosus--a 5-yr prospective study, Rheumatology (Oxford) 43 (2004) 1039-1044.

[110] M. Vadacca, D. Margiotta, D. Sambataro, F. Buzzulini, M. Lo Vullo, A. Rigon, et al., BAFF/APRIL pathway in Sjogren syndrome and systemic lupus erythematosus: relationship with chronic inflammation and disease activity, Reumatismo 62 (2010) 259-265.

[111] E.M. Ginzler, D.J. Wallace, J.T. Merrill, R.A. Furie, W. Stohl, W.W. Chatham, et al., Disease control and safety of belimumab plus standard therapy over 7 years in patients with systemic lupus erythematosus, J Rheumatol 41 (2014) 300-309.

[112] V. Strand, R.A. Levy, R. Cervera, M.A. Petri, H. Birch, W.W. Freimuth, et al., Improvements in health-related quality of life with belimumab, a B-lymphocyte stimulator-specific inhibitor, in patients with autoantibody-positive systemic lupus erythematosus from the randomised controlled BLISS trials, Ann Rheum Dis 73 (2014) 838-844.

[113] L. Iaccarino, S. Bettio, R. Reggia, M. Zen, M. Frassi, L. Andreoli, et al., Effects of Belimumab on Flare Rate and Expected Damage Progression in Patients With Active Systemic Lupus Erythematosus, Arthritis Care Res 69 (2017) 115-123.

[114] M. Mosca, C. Tani, M. Aringer, Withdrawal of therapy in non-renal systemic lupus erythematosus: is this an achievable goal, Clin Exp Rheumatol 31 (2013) 71-74.

[115] M.B. Urowitz, M. Feletar, I.N. Bruce, D. Ibañez, D.D. Gladman, Prolonged remission in systemic lupus erythematosus, J Rheumatol 32 (2005) 1467-1472.

[116] G. Moroni, S. Longhi, E. Giglio, P. Messa, C. Ponticelli, What happens after complete withdrawal of therapy in patients with lupus nephritis, Clin Exp Rheumatol 31 (2013) S75-S81.

[117] G. Moroni, F. Raffiotta, C. Ponticelli, Remission and withdrawal of therapy in lupus nephritis, J Nephrol 29 (2016) 559-565.

[118] G. Moroni, M. Gatto, F. Raffiotta, V. Binda, E. Frangou, L. Lightstone, et al., Can we withdraw immunosuppressants in patients with lupus nephritis in remission? An expert debate, Autoimmun Rev 17 (2018) 11-18.

[119] D.J. Wallace, W. Stohl, R.A. Furie, J.R. Lisse, J.D. McKay, J.T. Merrill, et al., A phase II, randomized, double-blind, placebo-controlled, dose-ranging study of belimumab in patients with active systemic lupus erythematosus, Arthritis Rheum 61 (2009) 1168-1178.

[120] D.A. Roth, A. Thompson, Y. Tang, A.E. Hammer, C.T. Molta, D. Gordon, Elevated BLyS levels in patients with systemic lupus erythematosus: Associated factors and responses to belimumab, Lupus 25 (2016) 346-354.
[121] Gerosa M, Meroni PL, Erkan D. Recognition and management of antiphospholipid syndrome. Curr Opin Rheumatol 201;28(1):51-9

[122] S. Miyakis, M.D. Lockshin, T. Atsumi, D.W. Branch, R.L. Brey, R. Cervera, et al., International consensus statement on an update of the classification criteria for definite antiphospholipid syndrome (APS), J Thromb Haemost 4 (2006) 295-306.

[123] P.L. Meroni, C.B. Chighizola, F. Rovelli, M. Gerosa, Antiphospholipid syndrome in 2014: more clinical manifestations, novel pathogenic players and emerging biomarkers, Arthritis Res Ther 16 (2014) 209.

[124] C.B. Chighizola, M. Gerosa, P.L. Meroni, New tests to detect antiphospholipid antibodies: anti-dmanin I beta2 glycorpotein I antibodies, Curr Rheumatol Rep 16 (2014) 402.

[125] A. Agostinis, P. Durigutto, B. Sblattero, M.O. Borghi, C. Grossi, F. Guida, et al., Tedesco F. A non-complement-fixing antibody to 2 glycoprotein I as a novel therapy for antiphospholipid syndrome, Blood 123 (2014) 3478-3487.

[126] Y. Ioannou, Z. Romay-Penabad, C. Pericleous, et al., In vivo inhibition of antiphospholipid antibody-induced pathogenicity utilizing the antigenic target peptide domain I of beta2-glycoprotein I: proof of concept, J Thromb Haemost 7 (2009) 833-842.

[127] C. Pericleous, P. Ruiz-Limo', Z. Romay-Penabad, Marı́n A. Carrera, A. Garza-Garcia, L. Murfitt, et al., Proof-of-concept study demonstrating the pathogenicity of affinity-purified IgG antibodies directed to domain I of b2-glycoprotein I in a mouse model of anti-phospholipid antibody-induced thrombosis, Rheumatology 54 (2015) 722-727.

[128] Andreoli L, Nalli C, Motta M, Norman GL, Shums Z, Encabo S, Binder WL, Nuzzo M, Frassi M, Lojacono A, Avcin T, Meroni PL, Tincani A. Anti- $\beta_{2}$-glycoprotein I IgG antibodies from 1-year-old healthy children born to mothers with systemic autoimmune diseases preferentially target domain 4/5: might it be the reason for their 'innocent' profile?

[129] L. Andreoli, C.B. Chighizola, C. Nalli, M. Gerosa, M.O. Borghi, F. Pregnolato, et al., Clinical characterization of antiphospholipid syndrome by detection of IgG antibodies against $\beta 2$-glycoprotein i domain 1 and domain 4/5: ratio of anti-domain 1 to anti-domain $4 / 5$ as a useful new biomarker for antiphospholipid syndrome, Arthritis Rheum 67 (2015) 2196-2204.

[130] M. Radin, I. Cecchi, D. Roccatello, P.L. Meroni, S. Sciascia, Prevalence and thrombotic risk assessment of anti-beta2 glycoprotein I domain I antibodies: a systematic review, Sem Thromb Haemost (2017).

[131] T. Iwaniec, M.P. Kaczor, M. Celińska-Löwenhoff, S. Polański, J. Musiał, Clinical significance of anti-domain $1 \beta 2$-glycoprotein I antibodies in antiphospholipid syndrome, Thromb Res 153 (2017 May) 90-94.

[132] J. Nojima, Y. Motoki, K. Hara, T. Sakata, H. Tsuneoka, K. Ichihara, Detection of antibodies against domain 1 of $\beta 2$-glycoprotein I is key in predicting thromboembolic complications in patients with systemic lupus erythematosus, Thromb Res 153 (2017 May) 83-84.

[133] C. Pericleous, I. Ferreira, O. Borghi, F. Pregnolato, T. McDonnell, A. Garza-Garcia, et al., Measuring IgA Anti-ß2-Glycoprotein I and IgG/IgA Anti-Domain I Antibodies Adds Value to Current Serological Assays for the Antiphospholipid Syndrome, PLoS One 11 (6) (2016 Jun 2), e0156407.

[134] K. Oku, O. Amengual, M. Kato, T. Bohgaki, T. Horita, S. Yasuda, et al., Significance of fully automated tests for the diagnosis of antiphospholipid syndrome, Thromb Res 146 (2016 Oct) 1-6.

[135] V. Pengo, A. Ruffatti, M. Tonello, A. Hoxha, E. Bison, G. Denas, et al., Antibodies to Domain 4/5 (Dm4/5) of $\beta 2$-Glycoprotein 1 ( $\beta 2 \mathrm{GP} 1$ ) in different antiphospholipid (aPL) antibody profiles, Thromb Res 136 (2015) 161-163.

[136] E. Akhter, Z. Shums, G.L. Norman, W. Binder, H. Fang, M. Petri, Utility of antiphosphatidylserine/prothrombin and IgA antiphospholipid assays in systemic lupus erythematosus, J Rheumatol 40 (3) (2013) 282-286.

[137] A.S. De Craemer, J. Musial, K.M. Devreese, Role of anti-domain 1-b2 glycoprotein I antibodies in the diagnosis and risk stratification of ntiphospholipid syndrome, J Thromb Haemost 14 (9) (2016) 1779-1787.

[138] M.L. Bertolaccini, O. Amengual, L. Andreoli, T. Atsumi, C.B. Chighizola, R. Forastiero, et al., 14th International Congress on Antiphospholipid Antibodie Task Force. Report on antiphospholipid syndrome laboratory diagnostics and trends, Autoimmun Rev 13 (9) (2014 Sep) 917-930.

[139] S. Sciascia, G. Sanna, V. Murru, D. Roccatello, M.A. Khamashta, M.L. Bertolac cini, Anti-prothrombin (aPT) and anti-phosphatidylserine/prothrombin (aPS/PT) antibodies and the risk of thrombosis in the antiphospholipid syndrome. A systematic review, Thromb Haemost 111 (2) (2014 Feb) 354-364.

[140] P. Žigon, S. Čučnik, A. Ambrožič, T. Kveder, S.S. Šemrl, B. Rozman, et al., Detection of antiphosphatidylserine/prothrombin antibodies and their potential diagnostic value, Clin Dev Immunol 2013 (2013), 724592.

[141] O. Amengual, R. Forastiero, M. Sugiura-Ogasawara, K. Otomo, K. Oku, C. Favas, et al., Evaluation of phosphatidylserine-dependent antiprothrombin antibody testing for the diagnosis of antiphospholipid syndrome: results of an internationa multicentre study, Lupus 26 (3) (2017 Mar) 266-276.

[142] A. Hoxha, A. Ruffatti, M. Tonello, A. Bontadi, E. Salvan, A. Banzato, et al., Antiphosphatidylserine/prothrombin antibodies in primary antiphospholipid syndrome, Lupus 21 (7) (2012 Jun) 787-789.

[143] G. Espinosa, R. Cervera, Current treatment of antiphospholipid syndrome: lights and shadows, Nat Rev Rheumatol 11 (10) (2015 Oct) 586-596.

[144] Conti F, Capozzi A, Truglia S, Lococo E, Longo A, Misasi R, Alessandri C, Valesini G, Sorice M. The mosaic of "seronegative" antiphospholipid syndrome. J Immunol Res 2014;2014:389601

[145] H. Shi, H. Zheng, Y.F. Yin, Q.Y. Hu, J.L. Teng, Y. Sun, et al., Antiphosphatidylserine/prothrombin antibodies (aPS/PT) as potential diagnostic markers and risk predictors of venous thrombosis and obstetric complications in antiphospholipid syndrome, Clin Chem Lab Med (2017 Nov 22).

[146] C.P. Mavragani, A. Nezos, H.M. Moutsopoulos, New advances in the classification, pathogenesis and treatment of Sjogren's syndrome, Curr Opin Rheumatol 25 
[147] G. Nocturne, X. Mariette, Advances in understanding the pathogenesis of primary Sjögren's syndrome, Nat Rev Rheumatol 9 (9) (2013 Sep) 544-556.

[148] L. Quartuccio, M. Isola, C. Baldini, R. Priori, E. Bartoloni Bocci, F. Carubbi, et al., Biomarkers of lymphoma in Sjögren's syndrome and evaluation of the lymphoma risk in prelymphomatous conditions: results of a multicenter study, J Autoimmun 51 (2014 Jun) 75-80.

[149] P. Brito-Zerón, M. Ramos-Casals, A. Bove, J. Sentis, J. Font, Predicting adverse outcomes in primary Sjogren's syndrome: identification of prognostic factors, Rheumatology (Oxford) 46 (8) (2007 Aug) 1359-1362.

[150] C.G. Vinuesa, S.G. Tangye, B. Moser, C.R. Mackay, Follicular B helper T cells in antibody responses and autoimmunity, Nat Rev Immunol 5 (11) (2005 Nov) 853-865.

[151] C. Havenar-Daughton, CXCL13 is a plasma biomarker of germinal center activity, Proc Natl Acad Sci U S A 113 (10) (2016 Mar 8) 2702-2707.

[152] J. Groom, S.L. Kalled, A.H. Cutler, C. Olson, S.A. Woodcock, P. Schneider, et al., Association of BAFF/BLyS overexpression and altered B cell differentiation with Sjögren's syndrome, J Clin Invest 109 (1) (2002 Jan) 59-68.

[153] A. Hansen, P.E. Lipsky, T. Dörner, B cells in Sjögren's syndrome: indications for disturbed selection and differentiation in ectopic lymphoid tissue, Arthritis Res Ther 9 (4) (2007) 218 .

[154] G. Nocturne, R. Seror, O. Fogel, R. Belkhir, S. Boudaoud, A. Saraux, et al., CXCL13 and CCL11 Serum Levels and Lymphoma and Disease Activity in Primary Sjögren's Syndrome, Arthritis Rheum 67 (12) (2015 Dec) 3226-3233.

[155] J.E. Gottenberg, R. Seror, C. Miceli-Richard, J. Benessiano, V. Devauchelle-Pensec, P. Dieude, et al., Serum levels of beta2-microglobulin and free light chains of immunoglobulins are associated with systemic disease activity in primary Sjögren's syndrome. Data at enrollment in the prospective ASSESS cohort, PLoS One 8 (5) (2013 May 24), e59868

[156] A.P. Risselada, M.F. Looije, A.A. Kruize, J.W. Bijlsma, J.A. van Roon, The role of ectopic germinal centers in the immunopathology of primary Sjögren's syndrome: a systematic review, Semin Arthritis Rheum 42 (4) (2013 Feb) 368-376.

[157] M.B. Nishishinya, C.A. Pereda, S. Muñoz-Fernández, J.M. Pego-Reigosa, I. Rúa-Figueroa, J.L. Andreu, et al., Identification of lymphoma predictors in patients with primary Sjögren's syndrome: a systematic literature review and meta-analysis, Rheumatol Int 35 (1) (2015 Jan) 17-26.

[158] A. Nishikawa, K. Suzuki, Y. Kassai, Y. Gotou, M. Takiguchi, T. Miyazaki, et al., Identification of definitive serum biomarkers associated with disease activity in primary Sjögren's syndrome, Arthritis Res Ther 18 (1) (2016 May 14) 106.

[159] G.M. Verstappen, P.M. Meiners, O.B.J. Corneth, A. Visser, S. Arends, W.H. Abdulahad, et al., Attenuation of Follicular Helper T Cell-Dependent B Cell Hyperactivity by Abatacept Treatment in Primary Sjögren's Syndrome, Arthritis Rheum 69 (9) (2017 Sep) 1850-1861.

[160] A. Hansen, P.E. Lipsky, T. Dörner, B cells in Sjögren's syndrome: indications for disturbed selection and differentiation in ectopic lymphoid tissue, Arthritis Res Ther 9 (4) (2007) 218.
[161] D. Cornec, S. Costa, V. Devauchelle-Pensec, S. Jousse-Joulin, P. Marcorelles, J.M. Berthelot, et al., Blood and salivary-gland BAFF-driven B-cell hyperactivity is associated to rituximab inefficacy in primary Sjögren's syndrome, J Autoimmun 67 (2016 Feb) 102-110.

[162] R. Seror, G. Nocturne, T. Lazure, H. Hendel-Chavez, F. Desmoulins, R. Belkhir, et al., Low numbers of blood and salivary natural killer cells are associated with a better response to belimumab in primary Sjögren's syndrome: results of the BELISS study, Arthritis Res Ther 17 (2015 Sep 4) 241.

[163] E. Theander, L. Vasaitis, E. Baecklund, G. Nordmark, G. Warfvinge, R. Liedholm, et al., Lymphoid organisation in labial salivary gland biopsies is a possible predictor for the development of malignant lymphoma in primary Sjögren's syndrome, Ann Rheum Dis 70 (8) (2011 Aug) 1363-1368.

[164] M. Bombardieri, F. Barone, F. Humby, S. Kelly, M. McGurk, P. Morgan, et al., Activation-induced cytidine deaminase expression in follicular dendritic cell networks and interfollicular large B cells supports functionality of ectopic lymphoid neogenesis in autoimmune sialoadenitis and MALT lymphoma in Sjögren's syndrome, J Immunol 179 (7) (2007 Oct 1) 4929-4938.

[165] F. Carubbi, A. Alunno, P. Cipriani, P. Di Benedetto, P. Ruscitti, O. Berardicurti, et al., Giacomelli R. Is minor salivary gland biopsy more than a diagnostic tool in primary Sjögren's syndrome? Association between clinical, histopathological, and molecular features: a retrospective study, Semin Arthritis Rheum 44 (3) (2014 Dec) 314-324.

[166] A.P. Risselada, A.A. Kruize, R. Goldschmeding, F.P. Lafeber, J.W. Bijlsma, J.A. van Roon, The prognostic value of routinely performed minor salivary gland assessments in primary Sjögren's syndrome, Ann Rheum Dis 73 (8) (2014 Aug) $1537-1540$

[167] E.A. Haacke, B. van der Vegt, A. Vissink, F.K.L. Spijkervet, H. Bootsma, F.G.M. Kroese, Germinal centres in diagnostic labial gland biopsies of patients with primary Sjögren's syndrome are not predictive for parotid MALT lymphoma development, Ann Rheum Dis 76 (10) (2017 Oct) 1781-1784.

[168] B.A. Fisher, R. Jonsson, T. Daniels, M. Bombardieri, R.M. Brown, P. Morgan, et al., Sjögren's histopathology workshop group (appendix) from ESSENTIAL (EULAR Sjögren's syndrome study group). Standardisation of labial salivary gland histopathology in clinical trials in primary Sjögren's syndrome, Ann Rheum Dis 76 (7) (2017 Jul) 1161-1168.

[169] K. De Boeck, C. Castellani, J.S. Elborn, E.C.F.S. Board, Medical consensus, guidelines, and position papers: a policy for the ECFS, J Cyst Fibros 13 (5) (2014 Sep) 495-498.

[170] B. Foroutan, Personalized Medicine: A Review with Regard to Biomarkers, J Bioequiv Availab 7 (6) (2015) 244-256.

[171] S.J. Aronson, H.L. Rehm, Building the foundation for genomics in precision medicine, Nature 526 (2015) 336-342.

[172] J.M. Anaya, C. Duarte-Rey, J.C. Sarmiento-Monroy, D. Bardey, J. Castiblanco, A. Rojas-Villarraga, Personalized medicine. Closing the gap between knowledge and clinical practice, Autoimmun Rev 15 (8) (2016 Aug) 833-842. 